Supporting Information

\title{
Optical Fingerprints of Proteases and Their Inhibited Complexes Provided by Differential Cross-Reactivity of Fluorophore-Labeled Single-Stranded DNA
}

Shunsuke Tomita, ${ }^{* \dagger \dagger}$ Hiroka Sugai,${ }^{\dagger}$ Masahiro Mimura, ${ }^{\dagger} \S$ Sayaka Ishihara,${ }^{\dagger}$ Kentaro Shiraki, ${ }^{\S}$ and

$$
\text { Ryoji Kurita*,†, } \$
$$

$\dagger$ Biomedical Research Institute, National Institute of Advanced Industrial Science and Technology, and DAILAB, 1-1-1 Higashi, Tsukuba, Ibaraki 305-8566, Japan

*DBT-AIST International Laboratory for Advanced Biomedicine (DAILAB), DBT-AIST International Center for Translational \& Environmental Research (DAICENTER), National Institute of Advanced Industrial Science and Technology, 1-1-1 Higashi, Tsukuba, Ibaraki 305-8566, Japan

$\S$ Faculty of Pure and Applied Sciences, University of Tsukuba, 1-1-1 Tennodai, Tsukuba, Ibaraki 3058573, Japan

\footnotetext{
*E-mail: s.tomita@aist.go.jp

*E-mail: r.kurita@aist.go.jp
} 


\section{Experimental details}

\section{Materials}

ssDNAs labeled with and without TAMRA at the 3'-terminus were synthesized and purified by Eurofins Genomics (Ebersberg, Germany). Carboxypeptidase A from bovine pancreas (Car), thrombin from bovine plasma (ThrB), elastase from porcine pancreas (Ela), $\alpha$-chymotrypsin from bovine pancreas (Chy), proteinase K from Tritirachium album (Pro), papain from papaya latex (Pap), trypsin from bovine pancreas (TryB), trypsin from porcine pancreas (TryP), $\alpha_{1}$-antitrypsin from human plasma (ATT), antithrombin III from human plasma (AThr), 2-morpholinoethanesulfonic acid (MES), 3morpholinopropanesulfonic acid (MOPS), and polyethylene glycol with an average molecular weight of 10000 (PEG10000) were purchased from Sigma-Aldrich, Co., LLC. (St. Louis, MO, USA). Thrombin from human plasma (ThrH) was purchased from Haematologic Technologies Inc. (Essex, VT, USA). Benzoyl-L-arginine $p$-nitroanilide monohydrochloride (L-BAPA) and $t$-butyloxycarbonyl-L-valyl-Lprolyl-L-arginine 4-methylcoumaryl-7-amide (Boc-Val-Pro-Arg-MCA) were purchased from the Peptide Institute (Osaka, Japan). Human serum was purchased from Cosmo Bio (Tokyo, Japan). Dimethyl sulfoxide (DMSO) was purchased from Wako Pure Chemical Ind. (Osaka, Japan).

\section{Circular dichroism measurements}

Circular dichroism (CD) experiments were performed on a J-720 spectropolarimeter (Japan Spectroscopic Co., Ltd.). Solutions containing $50 \mu \mathrm{M}$ ssDNAs without TAMRA modification in $20 \mathrm{mM}$ MOPS buffer $(\mathrm{pH}=7.4)$ or $20 \mathrm{mM}$ MES buffer $(\mathrm{pH}=5.4)$ were placed in a $1 \mathrm{~mm}$ quartz cell, and the spectra were recorded at $30{ }^{\circ} \mathrm{C}$. The $\mathrm{CD}$ spectra of the samples were corrected by subtracting the corresponding spectra of the buffers in the absence of ssDNA.

\section{Fluorescence measurements}

The concentration of proteases and the protease inhibitors was determined from their absorbance on a NanoDrop 1000 spectrophotometer (Thermo Scientific, Inc.) using the extinction coefficients shown in Table S1. Fluorescence measurements were performed on a Cytation5 Imaging Reader (BioTek Instruments, Inc.). Solutions $(200 \mu \mathrm{L})$ containing $20 \mathrm{nM}$ TAMRA-modified ssDNAs and 0-50 $\mu \mathrm{g} / \mathrm{mL}$ Pap in $20 \mathrm{mM}$ MOPS buffer ( $\mathrm{pH}=7.4)$ or $20 \mathrm{mM}$ MES buffer $(\mathrm{pH}=5.4)$ were prepared in each well of 
a 96-well NBS ${ }^{\mathrm{TM}}$ black microplate (Corning Inc.) using a PIPETMAX liquid handling system (Gilson Inc.). After incubation $\left(30^{\circ} \mathrm{C}, 10 \mathrm{~min}\right)$, the fluorescence intensity was recorded at $30^{\circ} \mathrm{C}$ at $\lambda_{\mathrm{ex}} / \lambda_{\mathrm{em}}=530$ $\mathrm{nm} / 570-700 \mathrm{~nm}$.

\section{Fingerprint-based sensing}

Aliquots $(180 \mu \mathrm{L})$ of the TAMRA-modified ssDNAs $(22.2 \mathrm{nM})$ in $22.2 \mathrm{mM}$ MOPS buffer $(\mathrm{pH}=7.4)$ or 22.2 mM MES buffer ( $\mathrm{pH}=5.4$ ) were deposited in the wells of a 96-well plate using a PIPETMAX system. After incubation $\left(30{ }^{\circ} \mathrm{C}, 10 \mathrm{~min}\right)$, the fluorescence intensity was recorded in two different channels $\left(\mathrm{Ch} 1: \lambda_{\mathrm{ex}} / \lambda_{\mathrm{em}}=535 / 580 ; \mathrm{Ch} 2: \lambda_{\mathrm{ex}} / \lambda_{\mathrm{em}}=525 / 595\right)$. Subsequently, aliquots $(20 \mu \mathrm{L})$ of the analytes in distilled water were added to each well, and the fluorescence intensity was recorded after incubation $\left(30{ }^{\circ} \mathrm{C}, 10 \mathrm{~min}\right.$ ). When detecting complexes between $\mathrm{ThrH}$ and AThr, analytes were prepared by preincubating $\mathrm{ThrH}$ with/without AThr for $120 \mathrm{~min}$ at $25{ }^{\circ} \mathrm{C}$ in a solution containing $1.0 \mathrm{mg} / \mathrm{mL}$ PEG10000, $30 \mathrm{mM}$ MOPS ( $\mathrm{pH}=7.0$ ) before human serum was added. For the sensing, 1\% human serum and $0.1 \mathrm{mg} / \mathrm{mL}$ PEG10000 with $35 \mathrm{mM}$ MOPS $(\mathrm{pH}=7.4)$ or $35 \mathrm{mM}$ MES (pH = 5.4) and a 384-well $\mathrm{NBS}^{\mathrm{TM}}$ black plate (Corning Inc.) were used as solvents and the detection plate, respectively. The final concentration of the TAMRA-modified ssDNAs was $20 \mathrm{nM}$. For doubling the dataset by hybridization, 4 equiv of ssDNAs $(5 \mu \mathrm{L})$ complementary to the corresponding TAMRA-modified ssDNAs (Table S7) were added to the mixtures $(120 \mu \mathrm{L})$ of ssDNA-TAMRAs $(20 \mathrm{nM})$ and proteases $(10 \mu \mathrm{g} / \mathrm{mL})$ with $20 \mathrm{mM}$ MOPS buffer $(\mathrm{pH}=7.4)$, before the fluorescence intensity was recorded after incubation $\left(30^{\circ} \mathrm{C}, 10 \mathrm{~min}\right)$. This process was repeated six or eleven times to generate a training data matrix. This training data matrix was processed by linear discriminant analysis (LDA) and hierarchical clustering analysis (HCA) in SYSTAT 13 (Systat Inc.). For holdout testing, four fingerprints out of eleven for each analyte were randomly separated from the training data matrix and used as a test data matrix. The test data were classified in groups generated by the remaining training matrix according to their shortest Mahalanobis distances.

\section{Protease assay}

For TryB, aliquots $(361 \mu \mathrm{L})$ of TryB in $20 \mathrm{mM}$ MOPS buffer $(\mathrm{pH}=7.4)$ in the presence or absence of ATT were deposited in the wells of a 96-well clear microplate (AS ONE Corporation). After incubation (r.t., $5 \mathrm{~min})$, L-BAPA in DMSO $(19 \mu \mathrm{L})$ was added to each well, and the time course of the increase in absorbance at $410 \mathrm{~nm}$ was monitored using a Cytation5 Imaging Reader. The final concentrations were 
0-50 $\mu \mathrm{g} / \mathrm{mL}$ TryB, $2.5 \mathrm{mM}$ L-BAPA, $19 \mathrm{mM}$ MOPS buffer, and 5\% DMSO with or without $50 \mu \mathrm{g} / \mathrm{mL}$ ATT. For ThrH, $1010 \mathrm{nM}$ AThr was initially added to solutions containing 0-2020 nM ThrH, $1.0 \mathrm{mg} / \mathrm{mL}$ PEG10000, and $30.3 \mathrm{mM}$ MOPS ( $\mathrm{pH}=7.0$ ). The solutions were incubated for $120 \mathrm{~min}$ at $25^{\circ} \mathrm{C}$ to form a link via the ester bond between ThrH and AThr. ${ }^{1}$ The aliquots were then diluted 1000 -fold with solutions containing 1\% human serum and $30 \mathrm{mM}$ MOPS $(\mathrm{pH}=7.0)$, and deposited in the wells of a $96-$ well NBS ${ }^{\mathrm{TM}}$ black microplate $(96 \mu \mathrm{L})$, before $2.5 \mathrm{mM}$ Boc-Val-Pro-Arg-MCA in DMSO (4 $\mu \mathrm{L})$ was added to each well. The time course of the increase in fluorescence intensity was monitored at $\lambda_{\mathrm{ex}} / \lambda_{\mathrm{em}}=355 \mathrm{~nm} / 460 \mathrm{~nm}$. 


\section{Figures and Tables}

Table S1. Extinction coefficients of the proteins used in this study.

\begin{tabular}{lllcc}
\hline Analyte & Source & Abbr. & $\varepsilon\left(\mathrm{mg} / \mathrm{mL}^{-1} \mathrm{~cm}^{-1}\right.$ & Wavelength \\
\hline $\begin{array}{l}\text { Protease } \\
\text { carboxypeptidase A }\end{array}$ & bovine pancreas & Car & $1.88^{\mathrm{a}}$ & 278 \\
thrombin & bovine plasma & ThrB & $1.95^{\mathrm{a}}$ & 280 \\
elastase & porcine pancreas & Ela & $2.02^{\mathrm{a}}$ & 280 \\
$\begin{array}{l}\alpha \text {-chymotrypsin } \\
\text { proteinase K }\end{array}$ & bovine pancreas & Chy & $2.04^{\mathrm{a}}$ & 280 \\
papain & Tritirachium album & Pro & $1.42^{\mathrm{a}}$ & 280 \\
trypsin & papaya latex & Pap & $2.5^{\mathrm{a}}$ & 280 \\
trypsin & bovine pancreas & TryB & $1.54^{\mathrm{a}}$ & 280 \\
thrombin & porcine pancreas & TryP & $1.5^{\mathrm{a}}$ & 280 \\
Protease inhibitor & human plasma & ThrH & $1.95^{\mathrm{a}}$ & 280 \\
$\alpha_{1}$-antitrypsin & & & & \\
antithrombin III & human serum & ATT & $0.45^{\mathrm{b}}$ & 280 \\
\hline
\end{tabular}

${ }^{a}$ According to the supplier's data sheet. ${ }^{b}$ Determined based on ref. 2 . 
Table S2. Data set matrix of the fluorescence intensity ratios before and after addition of $50 \mu \mathrm{g} / \mathrm{mL}$ proteases $\left(I / I_{0}\right)$ generated from the DNA array. The rightmost column shows the training data (denoted by "-") and the test data with the results of the verification by the holdout test.

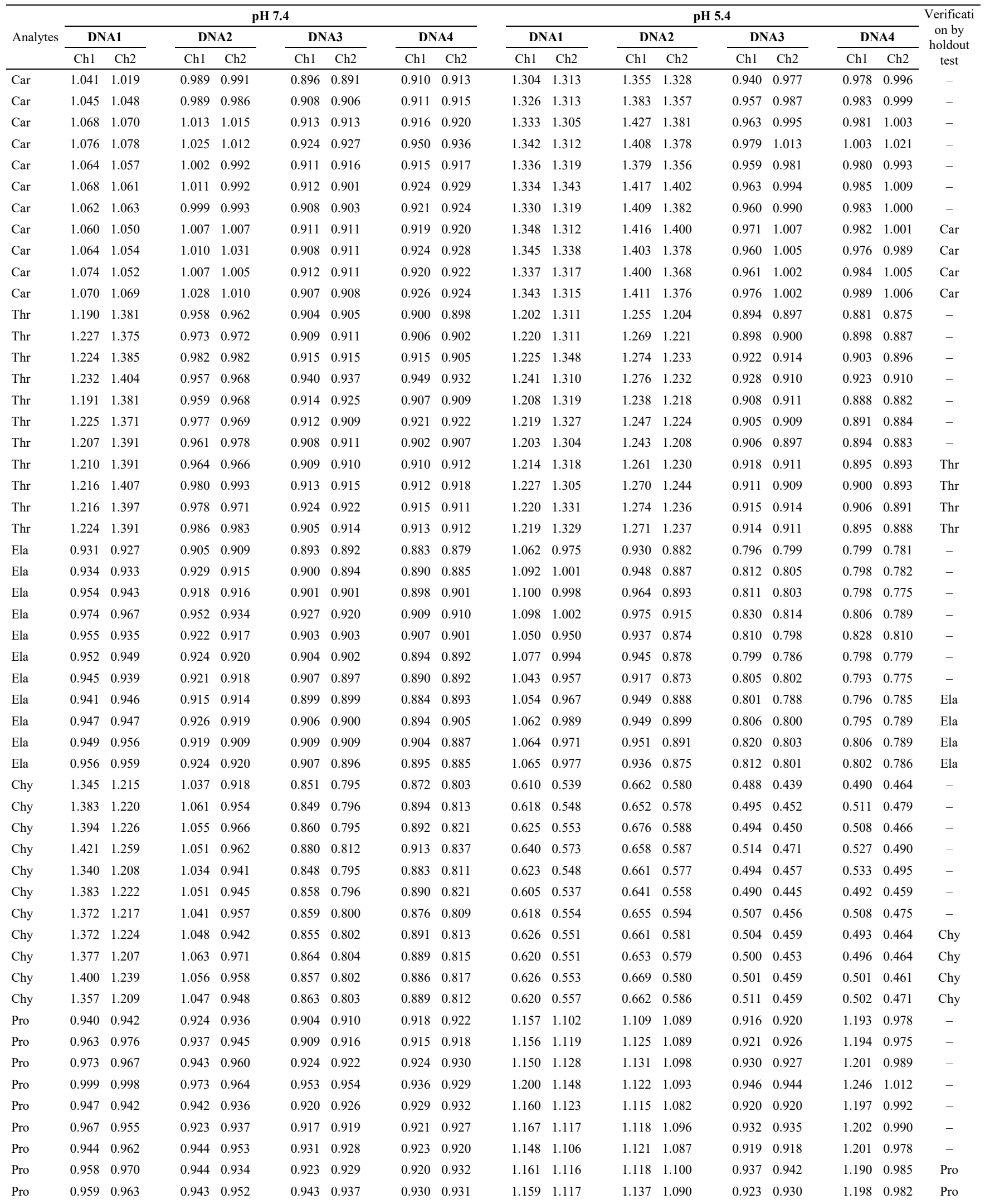




\begin{tabular}{|c|c|c|c|c|c|c|c|c|c|c|c|c|c|c|c|c|c|}
\hline Pro & 0.974 & 0.980 & 0.954 & 0.946 & 0.918 & 0.922 & 0.928 & 0.925 & 1.158 & 1.112 & 1.137 & 1.092 & 0.922 & 0.932 & 1.202 & 0.992 & Pro \\
\hline Pro & 0.955 & 0.980 & 0.938 & 0.945 & 0.925 & 0.925 & 0.924 & 0.927 & 1.153 & 1.128 & 1.122 & 1.093 & 0.921 & 0.927 & 1.197 & 0.981 & Pro \\
\hline Pap & 0.872 & 0.863 & 0.488 & 0.500 & 0.614 & 0.625 & 0.524 & 0.526 & 0.830 & 0.788 & 0.805 & 0.779 & 0.475 & 0.483 & 0.536 & 0.533 & - \\
\hline Pap & 0.884 & 0.871 & 0.483 & 0.494 & 0.616 & 0.625 & 0.530 & 0.532 & 0.831 & 0.807 & 0.814 & 0.772 & 0.480 & 0.490 & 0.530 & 0.525 & - \\
\hline Pap & 0.883 & 0.879 & 0.500 & 0.507 & 0.610 & 0.621 & 0.526 & 0.530 & 0.846 & 0.812 & 0.817 & 0.775 & 0.489 & 0.499 & 0.541 & 0.537 & - \\
\hline Pap & 0.920 & 0.898 & 0.481 & 0.493 & 0.630 & 0.639 & 0.540 & 0.544 & 0.867 & 0.812 & 0.818 & 0.776 & 0.491 & 0.501 & 0.546 & 0.545 & - \\
\hline Pap & 0.880 & 0.884 & 0.481 & 0.500 & 0.614 & 0.626 & 0.529 & 0.539 & 0.830 & 0.795 & 0.811 & 0.780 & 0.482 & 0.489 & 0.532 & 0.531 & - \\
\hline Pap & 0.890 & 0.883 & 0.485 & 0.498 & 0.620 & 0.623 & 0.531 & 0.535 & 0.854 & 0.806 & 0.810 & 0.768 & 0.486 & 0.497 & 0.537 & 0.529 & - \\
\hline Pap & 0.867 & 0.871 & 0.493 & 0.501 & 0.621 & 0.627 & 0.521 & 0.527 & 0.848 & 0.807 & 0.814 & 0.775 & 0.484 & 0.490 & 0.538 & 0.535 & - \\
\hline Pap & 0.887 & 0.874 & 0.494 & 0.501 & 0.626 & 0.635 & 0.530 & 0.532 & 0.847 & 0.810 & 0.813 & 0.778 & 0.481 & 0.487 & 0.531 & 0.538 & Pap \\
\hline Pap & 0.888 & 0.874 & 0.502 & 0.500 & 0.617 & 0.626 & 0.527 & 0.533 & 0.826 & 0.799 & 0.822 & 0.779 & 0.479 & 0.488 & 0.534 & 0.533 & Pap \\
\hline Pap & 0.888 & 0.876 & 0.492 & 0.500 & 0.611 & 0.618 & 0.523 & 0.528 & 0.920 & 0.873 & 0.807 & 0.772 & 0.490 & 0.499 & 0.538 & 0.533 & Pap \\
\hline Pap & 0.857 & 0.864 & 0.484 & 0.502 & 0.608 & 0.619 & 0.538 & 0.543 & 0.855 & 0.803 & 0.816 & 0.784 & 0.489 & 0.499 & 0.541 & 0.537 & Pap \\
\hline TryB & 1.245 & 1.103 & 1.006 & 0.917 & 0.828 & 0.788 & 0.850 & 0.786 & 0.650 & 0.572 & 0.686 & 0.620 & 0.535 & 0.491 & 0.536 & 0.497 & - \\
\hline TryB & 1.275 & 1.138 & 1.015 & 0.916 & 0.825 & 0.785 & 0.848 & 0.779 & 0.651 & 0.569 & 0.685 & 0.603 & 0.546 & 0.502 & 0.540 & 0.496 & - \\
\hline TryB & 1.273 & 1.134 & 1.025 & 0.934 & 0.836 & 0.799 & 0.845 & 0.790 & 0.657 & 0.599 & 0.692 & 0.607 & 0.549 & 0.505 & 0.551 & 0.517 & - \\
\hline TryB & 1.310 & 1.192 & 1.018 & 0.936 & 0.854 & 0.814 & 0.871 & 0.815 & 0.678 & 0.609 & 0.691 & 0.625 & 0.551 & 0.508 & 0.588 & 0.557 & - \\
\hline TryB & 1.243 & 1.134 & 1.005 & 0.928 & 0.838 & 0.796 & 0.852 & 0.800 & 0.654 & 0.583 & 0.668 & 0.603 & 0.542 & 0.499 & 0.527 & 0.495 & - \\
\hline TryB & 1.267 & 1.139 & 1.006 & 0.914 & 0.822 & 0.790 & 0.861 & 0.806 & 0.662 & 0.587 & 0.700 & 0.617 & 0.536 & 0.490 & 0.543 & 0.501 & - \\
\hline TryB & 1.265 & 1.142 & 1.012 & 0.913 & 0.821 & 0.791 & 0.845 & 0.795 & 0.649 & 0.567 & 0.719 & 0.631 & 0.537 & 0.496 & 0.536 & 0.499 & - \\
\hline TryB & 1.269 & 1.137 & 1.017 & 0.925 & 0.828 & 0.801 & 0.852 & 0.799 & 0.662 & 0.583 & 0.687 & 0.616 & 0.547 & 0.500 & 0.540 & 0.503 & TryB \\
\hline TryB & 1.269 & 1.142 & 1.012 & 0.929 & 0.832 & 0.787 & 0.852 & 0.792 & 0.654 & 0.585 & 0.688 & 0.615 & 0.541 & 0.496 & 0.537 & 0.498 & TryB \\
\hline TryB & 1.259 & 1.142 & 1.006 & 0.913 & 0.828 & 0.799 & 0.842 & 0.791 & 0.662 & 0.581 & 0.684 & 0.602 & 0.543 & 0.501 & 0.550 & 0.510 & TryB \\
\hline TryB & 1.250 & 1.154 & 1.022 & 0.924 & 0.827 & 0.795 & 0.851 & 0.804 & 0.655 & 0.582 & 0.692 & 0.612 & 0.543 & 0.499 & 0.532 & 0.496 & TryB \\
\hline TryP & 0.961 & 0.952 & 0.905 & 0.900 & 0.906 & 0.903 & 0.895 & 0.889 & 0.882 & 0.850 & 0.900 & 0.879 & 0.881 & 0.871 & 0.851 & 0.828 & - \\
\hline TryP & 0.966 & 0.963 & 0.938 & 0.935 & 0.911 & 0.908 & 0.898 & 0.892 & 0.877 & 0.851 & 0.887 & 0.876 & 0.864 & 0.867 & 0.853 & 0.841 & - \\
\hline TryP & 0.990 & 0.982 & 0.934 & 0.928 & 0.917 & 0.922 & 0.907 & 0.909 & 0.859 & 0.828 & 0.868 & 0.842 & 0.871 & 0.868 & 0.860 & 0.842 & - \\
\hline TryP & 0.996 & 0.990 & 0.949 & 0.938 & 0.938 & 0.936 & 0.916 & 0.919 & 0.890 & 0.860 & 0.907 & 0.883 & 0.882 & 0.882 & 0.883 & 0.873 & - \\
\hline TryP & 0.984 & 0.974 & 0.939 & 0.922 & 0.934 & 0.925 & 0.899 & 0.899 & 0.888 & 0.859 & 0.914 & 0.884 & 0.881 & 0.876 & 0.870 & 0.847 & - \\
\hline TryP & 0.979 & 0.963 & 0.925 & 0.928 & 0.921 & 0.921 & 0.908 & 0.907 & 0.892 & 0.860 & 0.912 & 0.893 & 0.874 & 0.880 & 0.860 & 0.845 & - \\
\hline TryP & 0.965 & 0.965 & 0.926 & 0.914 & 0.912 & 0.903 & 0.901 & 0.902 & 0.891 & 0.868 & 0.927 & 0.897 & 0.871 & 0.870 & 0.865 & 0.851 & - \\
\hline TryP & 0.986 & 0.976 & 0.928 & 0.929 & 0.929 & 0.920 & 0.909 & 0.905 & 0.890 & 0.857 & 0.937 & 0.912 & 0.887 & 0.883 & 0.871 & 0.857 & TryP \\
\hline TryP & 0.976 & 0.970 & 0.939 & 0.946 & 0.931 & 0.927 & 0.923 & 0.913 & 0.902 & 0.862 & 0.932 & 0.913 & 0.875 & 0.867 & 0.858 & 0.835 & TryP \\
\hline TryP & 0.990 & 0.966 & 0.948 & 0.925 & 0.923 & 0.919 & 0.910 & 0.912 & 0.904 & 0.859 & 0.928 & 0.898 & 0.886 & 0.882 & 0.899 & 0.871 & TryP \\
\hline TryP & 0.988 & 0.981 & 0.941 & 0.936 & 0.928 & 0.920 & 0.903 & 0.902 & 0.884 & 0.844 & 0.908 & 0.891 & 0.884 & 0.877 & 0.860 & 0.834 & TryP \\
\hline
\end{tabular}

Table S3. Effect of selected $\mathrm{pH}$ values on the classification accuracy of the DNA array for protein identification.

\begin{tabular}{cccc}
\hline \multicolumn{2}{c}{ Selected $\mathrm{pH}$} & $\begin{array}{c}\text { \% correct from } \\
\text { jackknife } \\
\text { classification }\end{array}$ & $\begin{array}{c}\text { \% correct } \\
\text { from holdout } \\
\text { test }\end{array}$ \\
\hline & $\mathrm{pH}=7.4$ & 100 & 100 \\
& & 94 & 97 \\
& 100 & 100 \\
\hline
\end{tabular}


Table S4. Data set matrix of the fluorescence intensity ratios before and after addition of varying concentrations of TryB and ATT $\left(I / I_{0}\right)$ generated from the DNA array. The rightmost column shows the training data (denoted by "-") and the test data with the results of the verification by the holdout test.

\begin{tabular}{|c|c|c|c|c|c|c|c|c|c|c|c|c|c|c|c|c|c|}
\hline \multirow{3}{*}{ Analytes } & \multicolumn{8}{|c|}{ pH 7.4} & \multicolumn{8}{|c|}{ pH 5.4} & \multirow{3}{*}{$\begin{array}{c}\text { Verification } \\
\text { by holdout } \\
\text { test }\end{array}$} \\
\hline & \multicolumn{2}{|c|}{ DNA1 } & \multicolumn{2}{|c|}{ DNA2 } & \multicolumn{2}{|c|}{ DNA3 } & \multicolumn{2}{|c|}{ DNA4 } & \multicolumn{2}{|c|}{ DNA1 } & \multicolumn{2}{|c|}{ DNA2 } & \multicolumn{2}{|c|}{ DNA3 } & \multicolumn{2}{|c|}{ DNA4 } & \\
\hline & Ch1 & Ch2 & Ch1 & Ch2 & Ch1 & Ch2 & Ch1 & Ch2 & Ch1 & $\mathrm{Ch} 2$ & Ch1 & $\mathrm{Ch} 2$ & Ch1 & $\mathrm{Ch} 2$ & Ch1 & $\mathrm{Ch} 2$ & \\
\hline $0 / 0$ & 0.903 & 0.891 & 0.881 & 0.880 & 0.874 & 0.855 & 0.865 & 0.870 & 0.872 & 0.885 & 0.855 & 0.866 & 0.871 & 0.859 & 0.869 & 0.872 & - \\
\hline $0 / 0$ & 0.886 & 0.920 & 0.885 & 0.861 & 0.885 & 0.868 & 0.870 & 0.853 & 0.865 & 0.897 & 0.860 & 0.880 & 0.880 & 0.872 & 0.877 & 0.873 & - \\
\hline $0 / 0$ & 0.899 & 0.887 & 0.879 & 0.874 & 0.879 & 0.886 & 0.891 & 0.885 & 0.858 & 0.871 & 0.879 & 0.882 & 0.880 & 0.894 & 0.869 & 0.865 & - \\
\hline $0 / 0$ & 0.898 & 0.907 & 0.881 & 0.901 & 0.879 & 0.885 & 0.887 & 0.877 & 0.882 & 0.915 & 0.853 & 0.861 & 0.899 & 0.904 & 0.873 & 0.873 & - \\
\hline $0 / 0$ & 0.909 & 0.901 & 0.889 & 0.876 & 0.883 & 0.877 & 0.881 & 0.874 & 0.857 & 0.821 & 0.842 & 0.841 & 0.877 & 0.891 & 0.879 & 0.869 & - \\
\hline $0 / 0$ & 0.880 & 0.889 & 0.887 & 0.905 & 0.890 & 0.885 & 0.882 & 0.881 & 0.869 & 0.880 & 0.808 & 0.839 & 0.880 & 0.877 & 0.859 & 0.861 & - \\
\hline $0 / 0$ & 0.871 & 0.898 & 0.859 & 0.895 & 0.878 & 0.871 & 0.874 & 0.864 & 0.843 & 0.880 & 0.810 & 0.823 & 0.876 & 0.875 & 0.881 & 0.861 & - \\
\hline $0 / 0$ & 0.924 & 0.899 & 0.893 & 0.864 & 0.889 & 0.887 & 0.885 & 0.888 & 0.867 & 0.851 & 0.817 & 0.811 & 0.878 & 0.877 & 0.858 & 0.868 & $0 / 0$ \\
\hline $0 / 0$ & 0.910 & 0.901 & 0.898 & 0.916 & 0.886 & 0.895 & 0.889 & 0.854 & 0.821 & 0.839 & 0.815 & 0.827 & 0.869 & 0.865 & 0.872 & 0.863 & $0 / 0$ \\
\hline $0 / 0$ & 0.897 & 0.906 & 0.888 & 0.853 & 0.876 & 0.876 & 0.869 & 0.866 & 0.870 & 0.846 & 0.800 & 0.812 & 0.869 & 0.871 & 0.857 & 0.873 & $0 / 0$ \\
\hline $0 / 0$ & 0.906 & 0.908 & 0.901 & 0.877 & 0.883 & 0.888 & 0.875 & 0.877 & 0.870 & 0.873 & 0.844 & 0.837 & 0.881 & 0.869 & 0.851 & 0.853 & $0 / 0$ \\
\hline $25 / 0$ & 0.892 & 0.885 & 0.875 & 0.880 & 0.882 & 0.869 & 0.867 & 0.878 & 1.506 & 1.356 & 1.193 & 1.059 & 0.857 & 0.830 & 0.935 & 0.886 & - \\
\hline $25 / 0$ & 0.889 & 0.867 & 0.893 & 0.902 & 0.893 & 0.892 & 0.883 & 0.890 & 1.553 & 1.443 & 1.157 & 1.089 & 0.876 & 0.852 & 0.974 & 0.886 & - \\
\hline $25 / 0$ & 0.922 & 0.914 & 0.902 & 0.887 & 0.898 & 0.888 & 0.898 & 0.901 & 1.561 & 1.435 & 1.196 & 1.112 & 0.885 & 0.855 & 0.985 & 0.909 & - \\
\hline $25 / 0$ & 0.907 & 0.908 & 0.910 & 0.902 & 0.898 & 0.898 & 0.907 & 0.896 & 1.549 & 1.423 & 1.194 & 1.106 & 0.879 & 0.853 & 0.963 & 0.898 & - \\
\hline $25 / 0$ & 0.917 & 0.907 & 0.896 & 0.885 & 0.885 & 0.906 & 0.873 & 0.881 & 1.493 & 1.362 & 1.158 & 1.070 & 0.862 & 0.838 & 0.951 & 0.889 & - \\
\hline $25 / 0$ & 0.919 & 0.949 & 0.899 & 0.881 & 0.893 & 0.888 & 0.880 & 0.879 & 1.541 & 1.431 & 1.175 & 1.106 & 0.862 & 0.847 & 0.932 & 0.869 & - \\
\hline $25 / 0$ & 0.910 & 0.867 & 0.901 & 0.901 & 0.888 & 0.901 & 0.891 & 0.904 & 1.533 & 1.409 & 1.146 & 1.102 & 0.869 & 0.855 & 0.948 & 0.908 & $25 / 0$ \\
\hline $25 / 0$ & 0.931 & 0.917 & 0.903 & 0.885 & 0.901 & 0.890 & 0.895 & 0.884 & 1.552 & 1.423 & 1.184 & 1.111 & 0.876 & 0.842 & 0.958 & 0.896 & $25 / 0$ \\
\hline $25 / 0$ & 0.893 & 0.916 & 0.896 & 0.883 & 0.884 & 0.897 & 0.885 & 0.877 & 1.551 & 1.370 & 1.139 & 1.070 & 0.870 & 0.838 & 0.950 & 0.890 & $25 / 0$ \\
\hline $25 / 0$ & 0.902 & 0.891 & 0.876 & 0.901 & 0.884 & 0.868 & 0.879 & 0.867 & 1.514 & 1.356 & 1.162 & 1.080 & 0.868 & 0.854 & 0.968 & 0.894 & $25 / 0$ \\
\hline $50 / 0$ & 0.873 & 0.891 & 0.887 & 0.871 & 0.876 & 0.883 & 0.887 & 0.879 & 1.498 & 1.418 & 1.189 & 1.077 & 0.862 & 0.800 & 0.920 & 0.860 & - \\
\hline $50 / 0$ & 0.898 & 0.905 & 0.885 & 0.900 & 0.889 & 0.891 & 0.876 & 0.888 & 1.525 & 1.362 & 1.168 & 1.090 & 0.878 & 0.810 & 0.942 & 0.890 & - \\
\hline $50 / 0$ & 0.888 & 0.909 & 0.896 & 0.903 & 0.877 & 0.896 & 0.902 & 0.882 & 1.507 & 1.344 & 1.188 & 1.082 & 0.867 & 0.830 & 0.934 & 0.857 & - \\
\hline $50 / 0$ & 0.889 & 0.894 & 0.916 & 0.904 & 0.895 & 0.882 & 0.887 & 0.900 & 1.545 & 1.402 & 1.224 & 1.117 & 0.882 & 0.818 & 0.941 & 0.882 & - \\
\hline $50 / 0$ & 0.941 & 0.888 & 0.900 & 0.906 & 0.881 & 0.889 & 0.889 & 0.883 & 1.470 & 1.419 & 1.201 & 1.115 & 0.874 & 0.825 & 0.953 & 0.893 & - \\
\hline $50 / 0$ & 0.893 & 0.895 & 0.893 & 0.910 & 0.874 & 0.885 & 0.885 & 0.907 & 1.535 & 1.406 & 1.187 & 1.107 & 0.876 & 0.830 & 0.938 & 0.878 & $50 / 0$ \\
\hline $50 / 0$ & 0.910 & 0.909 & 0.901 & 0.887 & 0.886 & 0.881 & 0.882 & 0.916 & 1.523 & 1.386 & 1.178 & 1.101 & 0.873 & 0.827 & 0.931 & 0.858 & $50 / 0$ \\
\hline $50 / 0$ & 0.892 & 0.902 & 0.875 & 0.859 & 0.889 & 0.889 & 0.879 & 0.869 & 1.534 & 1.373 & 1.154 & 1.052 & 0.862 & 0.840 & 0.935 & 0.856 & $25 / 0$ \\
\hline $50 / 0$ & 0.910 & 0.911 & 0.887 & 0.887 & 0.881 & 0.866 & 0.883 & 0.881 & 1.519 & 1.368 & 1.187 & 1.126 & 0.867 & 0.809 & 0.943 & 0.873 & $50 / 0$ \\
\hline $75 / 0$ & 0.879 & 0.906 & 0.881 & 0.896 & 0.869 & 0.877 & 0.876 & 0.878 & 1.460 & 1.345 & 1.211 & 1.100 & 0.865 & 0.815 & 0.905 & 0.825 & - \\
\hline $75 / 0$ & 0.928 & 0.911 & 0.903 & 0.882 & 0.870 & 0.890 & 0.873 & 0.879 & 1.483 & 1.342 & 1.209 & 1.120 & 0.888 & 0.819 & 0.904 & 0.832 & - \\
\hline $75 / 0$ & 0.893 & 0.897 & 0.900 & 0.904 & 0.887 & 0.867 & 0.882 & 0.877 & 1.465 & 1.347 & 1.196 & 1.068 & 0.865 & 0.793 & 0.899 & 0.861 & - \\
\hline $75 / 0$ & 0.905 & 0.895 & 0.918 & 0.908 & 0.892 & 0.915 & 0.891 & 0.887 & 1.535 & 1.342 & 1.218 & 1.149 & 0.886 & 0.825 & 0.909 & 0.831 & - \\
\hline $75 / 0$ & 0.901 & 0.919 & 0.904 & 0.906 & 0.887 & 0.896 & 0.892 & 0.893 & 1.489 & 1.379 & 1.219 & 1.087 & 0.878 & 0.805 & 0.897 & 0.836 & - \\
\hline $75 / 0$ & 0.912 & 0.873 & 0.893 & 0.883 & 0.885 & 0.893 & 0.883 & 0.899 & 1.505 & 1.339 & 1.195 & 1.086 & 0.861 & 0.813 & 0.902 & 0.842 & - \\
\hline $75 / 0$ & 0.912 & 0.892 & 0.868 & 0.885 & 0.881 & 0.880 & 0.865 & 0.880 & 1.516 & 1.399 & 1.178 & 1.087 & 0.865 & 0.797 & 0.892 & 0.832 & - \\
\hline $75 / 0$ & 0.933 & 0.865 & 0.876 & 0.890 & 0.889 & 0.885 & 0.884 & 0.885 & 1.453 & 1.345 & 1.177 & 1.084 & 0.870 & 0.829 & 0.898 & 0.828 & $75 / 0$ \\
\hline $75 / 0$ & 0.925 & 0.870 & 0.894 & 0.892 & 0.881 & 0.888 & 0.887 & 0.895 & 1.519 & 1.383 & 1.187 & 1.067 & 0.864 & 0.812 & 0.902 & 0.841 & $75 / 0$ \\
\hline $75 / 0$ & 0.882 & 0.899 & 0.880 & 0.857 & 0.892 & 0.873 & 0.886 & 0.886 & 1.460 & 1.326 & 1.194 & 1.064 & 0.862 & 0.805 & 0.899 & 0.834 & $75 / 0$ \\
\hline $75 / 0$ & 0.885 & 0.919 & 0.908 & 0.881 & 0.888 & 0.869 & 0.877 & 0.879 & 1.473 & 1.370 & 1.194 & 1.107 & 0.864 & 0.806 & 0.900 & 0.834 & $75 / 0$ \\
\hline $0 / 10$ & 0.893 & 0.902 & 0.854 & 0.844 & 0.844 & 0.847 & 0.803 & 0.795 & 0.504 & 0.500 & 0.387 & 0.390 & 0.417 & 0.425 & 0.265 & 0.258 & - \\
\hline $0 / 10$ & 0.871 & 0.885 & 0.849 & 0.838 & 0.842 & 0.843 & 0.816 & 0.809 & 0.533 & 0.554 & 0.396 & 0.411 & 0.413 & 0.400 & 0.273 & 0.281 & - \\
\hline $0 / 10$ & 0.900 & 0.892 & 0.845 & 0.836 & 0.844 & 0.839 & 0.814 & 0.794 & 0.527 & 0.522 & 0.406 & 0.411 & 0.426 & 0.421 & 0.274 & 0.278 & - \\
\hline $0 / 10$ & 0.929 & 0.909 & 0.862 & 0.868 & 0.863 & 0.861 & 0.804 & 0.807 & 0.533 & 0.535 & 0.417 & 0.403 & 0.430 & 0.425 & 0.279 & 0.278 & - \\
\hline $0 / 10$ & 0.890 & 0.916 & 0.850 & 0.857 & 0.861 & 0.859 & 0.818 & 0.799 & 0.536 & 0.550 & 0.421 & 0.402 & 0.414 & 0.413 & 0.275 & 0.286 & - \\
\hline $0 / 10$ & 0.913 & 0.919 & 0.848 & 0.819 & 0.849 & 0.842 & 0.809 & 0.808 & 0.514 & 0.519 & 0.391 & 0.401 & 0.415 & 0.411 & 0.267 & 0.277 & - \\
\hline $0 / 10$ & 0.895 & 0.855 & 0.848 & 0.843 & 0.837 & 0.838 & 0.796 & 0.807 & 0.559 & 0.562 & 0.415 & 0.410 & 0.413 & 0.417 & 0.272 & 0.269 & - \\
\hline $0 / 10$ & 0.924 & 0.928 & 0.861 & 0.845 & 0.842 & 0.841 & 0.799 & 0.789 & 0.532 & 0.530 & 0.402 & 0.398 & 0.428 & 0.429 & 0.268 & 0.275 & $0 / 10$ \\
\hline $0 / 10$ & 0.915 & 0.933 & 0.862 & 0.875 & 0.860 & 0.843 & 0.813 & 0.805 & 0.530 & 0.519 & 0.394 & 0.405 & 0.413 & 0.420 & 0.270 & 0.284 & $0 / 10$ \\
\hline
\end{tabular}




\begin{tabular}{|c|c|c|c|c|c|c|c|c|c|c|c|c|}
\hline $0 / 10$ & 0.908 & 0.904 & 0.841 & 0.842 & 0.839 & 0.831 & 0.796 & 0.815 & 0.525 & 0.519 & 0.394 & 0.387 \\
\hline $0 / 10$ & 0.912 & 0.889 & 0.858 & 0.851 & 0.843 & 0.849 & 0.798 & 0.818 & 0.510 & 0.517 & 0.396 & 0.400 \\
\hline $0 / 20$ & 0.894 & 0.877 & 0.828 & 0.806 & 0.836 & 0.840 & 0.794 & 0.794 & 0.363 & 0.380 & 0.293 & 0.281 \\
\hline $0 / 20$ & 0.885 & 0.871 & 0.808 & 0.823 & 0.836 & 0.834 & 0.806 & 0.807 & 0.374 & 0.380 & 0.310 & 0.304 \\
\hline $0 / 20$ & 0.899 & 0.893 & 0.834 & 0.832 & 0.849 & 0.838 & 0.797 & 0.800 & 0.396 & 0.406 & 0.304 & 0.297 \\
\hline $0 / 20$ & 0.910 & 0.859 & 0.835 & 0.818 & 0.845 & 0.843 & 0.801 & 0.806 & 0.374 & 0.389 & 0.313 & 0.301 \\
\hline $0 / 20$ & 0.886 & 0.884 & 0.850 & 0.834 & 0.852 & 0.848 & 0.810 & 0.822 & 0.392 & 0.414 & 0.308 & 0.308 \\
\hline $0 / 20$ & 0.908 & 0.913 & 0.811 & 0.823 & 0.839 & 0.842 & 0.804 & 0.810 & 0.387 & 0.403 & 0.312 & 0.309 \\
\hline $0 / 20$ & 0.911 & 0.893 & 0.817 & 0.820 & 0.852 & 0.829 & 0.789 & 0.805 & 0.407 & 0.408 & 0.326 & 0.315 \\
\hline $0 / 20$ & 0.939 & 0.893 & 0.809 & 0.812 & 0.850 & 0.832 & 0.809 & 0.809 & 0.406 & 0.399 & 0.296 & 0.293 \\
\hline $0 / 20$ & 0.921 & 0.922 & 0.833 & 0.819 & 0.848 & 0.845 & 0.814 & 0.818 & 0.381 & 0.377 & 0.296 & 0.293 \\
\hline $0 / 20$ & 0.863 & 0.863 & 0.799 & 0.799 & 0.835 & 0.845 & 0.803 & 0.796 & 0.386 & 0.401 & 0.306 & 0.301 \\
\hline $0 / 20$ & 0.913 & 0.921 & 0.833 & 0.794 & 0.844 & 0.851 & 0.805 & 0.799 & 0.386 & 0.391 & 0.286 & 0.281 \\
\hline $0 / 30$ & 0.910 & 0.901 & 0.785 & 0.779 & 0.837 & 0.831 & 0.763 & 0.779 & 0.308 & 0.304 & 0.239 & 0.228 \\
\hline $0 / 30$ & 0.909 & 0.924 & 0.800 & 0.781 & 0.846 & 0.843 & 0.789 & 0.782 & 0.340 & 0.349 & 0.271 & 0.266 \\
\hline $0 / 30$ & 0.917 & 0.945 & 0.798 & 0.783 & 0.851 & 0.851 & 0.790 & 0.800 & 0.315 & 0.319 & 0.243 & 0.236 \\
\hline $0 / 30$ & 0.909 & 0.899 & 0.801 & 0.816 & 0.859 & 0.848 & 0.786 & 0.800 & 0.317 & 0.318 & 0.241 & 0.255 \\
\hline $0 / 30$ & 0.947 & 0.924 & 0.800 & 0.789 & 0.849 & 0.841 & 0.793 & 0.788 & 0.307 & 0.313 & 0.259 & 0.244 \\
\hline $0 / 30$ & 0.914 & 0.942 & 0.788 & 0.781 & 0.842 & 0.837 & 0.800 & 0.785 & 0.318 & 0.317 & 0.244 & 0.233 \\
\hline $0 / 30$ & 0.908 & 0.916 & 0.806 & 0.800 & 0.846 & 0.852 & 0.787 & 0.779 & 0.335 & 0.323 & 0.261 & 0.249 \\
\hline $0 / 30$ & 0.933 & 0.924 & 0.797 & 0.776 & 0.830 & 0.836 & 0.783 & 0.789 & 0.331 & 0.325 & 0.245 & 0.242 \\
\hline $0 / 30$ & 0.929 & 0.939 & 0.808 & 0.796 & 0.841 & 0.851 & 0.793 & 0.798 & 0.319 & 0.317 & 0.245 & 0.239 \\
\hline $0 / 30$ & 0.917 & 0.902 & 0.772 & 0.798 & 0.834 & 0.831 & 0.776 & 0.770 & 0.315 & 0.311 & 0.255 & 0.233 \\
\hline $0 / 30$ & 0.916 & 0.902 & 0.795 & 0.786 & 0.849 & 0.842 & 0.792 & 0.790 & 0.315 & 0.301 & 0.246 & 0.240 \\
\hline $25 / 10$ & 0.872 & 0.909 & 0.898 & 0.877 & 0.885 & 0.871 & 0.876 & 0.877 & 1.348 & 1.254 & 1.035 & 0.972 \\
\hline $25 / 10$ & 0.924 & 0.920 & 0.880 & 0.896 & 0.884 & 0.904 & 0.897 & 0.879 & 1.394 & 1.260 & 1.086 & 1.007 \\
\hline $25 / 10$ & 0.926 & 0.909 & 0.903 & 0.894 & 0.902 & 0.888 & 0.896 & 0.896 & 1.392 & 1.253 & 1.064 & 0.983 \\
\hline $25 / 10$ & 0.929 & 0.929 & 0.903 & 0.928 & 0.909 & 0.904 & 0.901 & 0.918 & 1.421 & 1.279 & 1.112 & 1.022 \\
\hline $25 / 10$ & 0.921 & 0.918 & 0.931 & 0.909 & 0.891 & 0.895 & 0.906 & 0.890 & 1.422 & 1.278 & 1.089 & 0.996 \\
\hline $25 / 10$ & 0.908 & 0.935 & 0.913 & 0.905 & 0.900 & 0.902 & 0.880 & 0.874 & 1.411 & 1.280 & 1.053 & 0.966 \\
\hline $25 / 10$ & 0.882 & 0.918 & 0.894 & 0.904 & 0.887 & 0.902 & 0.872 & 0.879 & 1.435 & 1.278 & 1.095 & 1.026 \\
\hline $25 / 10$ & 0.898 & 0.918 & 0.875 & 0.882 & 0.888 & 0.891 & 0.875 & 0.868 & 1.367 & 1.255 & 1.060 & 0.989 \\
\hline $25 / 10$ & 0.932 & 0.905 & 0.904 & 0.915 & 0.896 & 0.908 & 0.903 & 0.914 & 1.415 & 1.249 & 1.062 & 1.016 \\
\hline $25 / 10$ & 0.914 & 0.935 & 0.892 & 0.884 & 0.888 & 0.890 & 0.898 & 0.888 & 1.393 & 1.245 & 1.071 & 0.970 \\
\hline $25 / 10$ & 0.922 & 0.912 & 0.899 & 0.906 & 0.902 & 0.882 & 0.874 & 0.879 & 1.385 & 1.254 & 1.067 & 0.961 \\
\hline $50 / 20$ & 0.931 & 0.911 & 0.908 & 0.897 & 0.923 & 0.931 & 0.902 & 0.901 & 1.337 & 1.239 & 1.076 & 1.009 \\
\hline $50 / 20$ & 0.937 & 0.909 & 0.911 & 0.915 & 0.879 & 0.889 & 0.898 & 0.884 & 1.346 & 1.236 & 1.060 & 0.964 \\
\hline $50 / 20$ & 0.914 & 0.950 & 0.884 & 0.874 & 0.870 & 0.863 & 0.884 & 0.864 & 1.277 & 1.141 & 1.030 & 0.940 \\
\hline $50 / 20$ & 0.915 & 0.955 & 0.908 & 0.879 & 0.890 & 0.892 & 0.905 & 0.905 & 1.328 & 1.224 & 1.034 & 0.935 \\
\hline $50 / 20$ & 0.904 & 0.904 & 0.920 & 0.895 & 0.891 & 0.907 & 0.880 & 0.870 & 1.308 & 1.180 & 1.065 & 0.954 \\
\hline $50 / 20$ & 0.923 & 0.903 & 0.901 & 0.910 & 0.891 & 0.891 & 0.875 & 0.866 & 1.309 & 1.164 & 1.020 & 0.956 \\
\hline $50 / 20$ & 0.931 & 0.955 & 0.907 & 0.907 & 0.906 & 0.895 & 0.876 & 0.889 & 1.300 & 1.188 & 1.023 & 0.961 \\
\hline $50 / 20$ & 0.928 & 0.919 & 0.915 & 0.898 & 0.898 & 0.896 & 0.895 & 0.873 & 1.305 & 1.185 & 1.038 & 0.944 \\
\hline $50 / 20$ & 0.943 & 0.927 & 0.898 & 0.901 & 0.897 & 0.895 & 0.884 & 0.893 & 1.322 & 1.253 & 1.076 & 0.961 \\
\hline $50 / 20$ & 0.918 & 0.952 & 0.894 & 0.910 & 0.900 & 0.908 & 0.882 & 0.884 & 1.362 & 1.224 & 1.052 & 0.978 \\
\hline $50 / 20$ & 0.926 & 0.927 & 0.899 & 0.886 & 0.881 & 0.879 & 0.878 & 0.868 & 1.307 & 1.158 & 1.041 & 0.974 \\
\hline $75 / 30$ & 0.962 & 0.933 & 0.915 & 0.900 & 0.932 & 0.918 & 0.934 & 0.949 & 1.333 & 1.265 & 1.064 & 0.965 \\
\hline $75 / 30$ & 0.939 & 0.957 & 0.897 & 0.895 & 0.898 & 0.909 & 0.874 & 0.869 & 1.290 & 1.101 & 1.017 & 0.966 \\
\hline $75 / 30$ & 0.915 & 0.947 & 0.888 & 0.872 & 0.900 & 0.890 & 0.875 & 0.889 & 1.293 & 1.151 & 1.028 & 0.927 \\
\hline $75 / 30$ & 0.958 & 0.930 & 0.893 & 0.885 & 0.885 & 0.881 & 0.895 & 0.903 & 1.252 & 1.154 & 1.032 & 0.945 \\
\hline $75 / 30$ & 0.931 & 0.913 & 0.892 & 0.890 & 0.892 & 0.897 & 0.872 & 0.861 & 1.254 & 1.190 & 1.032 & 0.956 \\
\hline $75 / 30$ & 0.940 & 0.978 & 0.897 & 0.907 & 0.894 & 0.901 & 0.864 & 0.864 & 1.265 & 1.152 & 0.998 & 0.885 \\
\hline $75 / 30$ & 0.939 & 0.942 & 0.891 & 0.881 & 0.882 & 0.880 & 0.852 & 0.868 & 1.248 & 1.127 & 1.022 & 0.902 \\
\hline $75 / 30$ & 0.949 & 0.943 & 0.901 & 0.880 & 0.895 & 0.901 & 0.864 & 0.866 & 1.241 & 1.121 & 1.017 & 0.931 \\
\hline $75 / 30$ & 0.933 & 0.938 & 0.892 & 0.893 & 0.899 & 0.915 & 0.876 & 0.862 & 1.281 & 1.178 & 1.049 & 0.950 \\
\hline $75 / 30$ & 0.939 & 0.934 & 0.898 & 0.898 & 0.898 & 0.897 & 0.867 & 0.861 & 1.289 & 1.130 & 1.036 & 0.947 \\
\hline $75 / 30$ & 0.942 & 0.947 & 0.899 & 0.882 & 0.885 & 0.885 & 0.879 & 0.873 & 1.240 & 1.125 & 1.024 & 0.940 \\
\hline $50 / 10$ & 0.913 & 0.918 & 0.914 & 0.917 & 0.912 & 0.925 & 0.920 & 0.921 & 1.504 & 1.360 & 1.158 & 1.047 \\
\hline $50 / 10$ & 0.913 & 0.945 & 0.888 & 0.921 & 0.895 & 0.901 & 0.886 & 0.897 & 1.398 & 1.344 & 1.113 & 1.035 \\
\hline $50 / 10$ & 0.900 & 0.890 & 0.879 & 0.909 & 0.877 & 0.891 & 0.858 & 0.869 & 1.428 & 1.309 & 1.109 & 1.006 \\
\hline $50 / 10$ & 0.928 & 0.921 & 0.892 & 0.892 & 0.896 & 0.894 & 0.897 & 0.891 & 1.427 & 1.296 & 1.122 & 1.033 \\
\hline $50 / 10$ & 0.915 & 0.924 & 0.920 & 0.891 & 0.893 & 0.893 & 0.891 & 0.887 & 1.426 & 1.299 & 1.150 & 1.002 \\
\hline $50 / 10$ & 0.899 & 0.955 & 0.893 & 0.902 & 0.892 & 0.887 & 0.886 & 0.884 & 1.427 & 1.307 & 1.135 & 1.014 \\
\hline
\end{tabular}

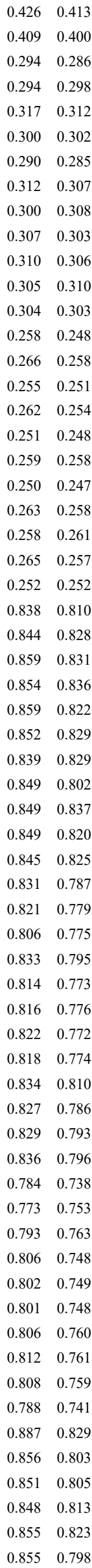

$0.271 \quad 0.272$

$\begin{array}{lll}0.193 & 0.193\end{array}$

$0.195 \quad 0.190$

$0.190 \quad 0.193$

$\begin{array}{ll}0.189 & 0.193\end{array}$

$0.191 \quad 0.190$

$\begin{array}{ll}0.195 & 0.191\end{array}$

$\begin{array}{ll}0.192 & 0.199\end{array}$

$0.189 \quad 0.194$

$\begin{array}{lll}0.192 & 0.193\end{array}$

$\begin{array}{ll}0.193 & 0.193\end{array}$

$\begin{array}{ll}0.187 & 0.189\end{array}$

$0.148 \quad 0.148$
0.165

$\begin{array}{ll}0.165 & 0.169\end{array}$

$\begin{array}{lll}0.154 & 0.158\end{array}$

$\begin{array}{ll}0.158 & 0.158\end{array}$

$\begin{array}{ll}0.152 & 0.152\end{array}$

$\begin{array}{ll}0.152 & 0.155\end{array}$

$\begin{array}{ll}0.158 & 0.158\end{array}$

$\begin{array}{ll}0.152 & 0.157\end{array}$

$\begin{array}{ll}0.156 & 0.157\end{array}$

$\begin{array}{ll}0.150 & 0.148\end{array}$

$\begin{array}{ll}0.151 & 0.153\end{array}$

$\begin{array}{lll}0.906 & 0.844\end{array}$

$\begin{array}{ll}0.906 & 0.835\end{array}$

$\begin{array}{ll}0.910 & 0.831\end{array}$

$\begin{array}{lll}0.922 & 0.858\end{array}$

$0.914 \quad 0.853$

$0.921 \quad 0.837$

$0.909 \quad 0.850$

$\begin{array}{lll}0.928 & 0.832\end{array}$

$\begin{array}{ll}0.933 & 0.844\end{array}$

$\begin{array}{ll}0.922 & 0.849\end{array}$

$0.912 \quad 0.838$

$\begin{array}{ll}0.870 & 0.788\end{array}$

$\begin{array}{ll}0.873 & 0.777\end{array}$

$\begin{array}{lll}0.853 & 0.778\end{array}$

$\begin{array}{ll}0.834 & 0.809\end{array}$

$\begin{array}{ll}0.849 & 0.765\end{array}$

$0.841 \quad 0.777$

$\begin{array}{ll}0.850 & 0.777\end{array}$

\begin{tabular}{ll}
$0.893 \quad 0.816$ \\
\hline
\end{tabular}

$\begin{array}{lll}0.857 & 0.809\end{array}$

$0.858 \quad 0.796$

$\begin{array}{ll}0.851 & 0.787\end{array}$

$\begin{array}{ll}0.820 & 0.755\end{array}$

$0.807 \quad 0.730$

$\begin{array}{ll}0.809 & 0.735\end{array}$

$\begin{array}{ll}0.804 & 0.731\end{array}$

$\begin{array}{ll}0.816 & 0.737\end{array}$

$\begin{array}{ll}0.796 & 0.719\end{array}$

$\begin{array}{ll}0.806 & 0.773\end{array}$

$\begin{array}{ll}0.815 & 0.760\end{array}$

$\begin{array}{ll}0.828 & 0.755\end{array}$

\begin{tabular}{ll}
$0.828 \quad 0.764$ \\
\hline
\end{tabular}

$\begin{array}{lll}0.804 & 0.742\end{array}$

$0.924 \quad 0.871$

$\begin{array}{ll}0.917 & 0.818\end{array}$

$\begin{array}{ll}0.898 & 0.822\end{array}$

$0.906 \quad 0.844$

$\begin{array}{ll}0.881 & 0.818\end{array}$

$0.881 \quad 0.834$
$0 / 10$ $0 / 10$

$-$ 


\begin{tabular}{|c|c|c|c|c|c|c|c|c|c|c|c|c|c|c|c|c|c|}
\hline $50 / 10$ & 0.917 & 0.921 & 0.910 & 0.917 & 0.902 & 0.888 & 0.906 & 0.913 & 1.424 & 1.270 & 1.098 & 1.020 & 0.856 & 0.811 & 0.911 & 0.835 & - \\
\hline $50 / 10$ & 0.919 & 0.910 & 0.902 & 0.898 & 0.882 & 0.881 & 0.872 & 0.880 & 1.428 & 1.301 & 1.133 & 1.055 & 0.855 & 0.799 & 0.907 & 0.835 & $50 / 10$ \\
\hline $50 / 10$ & 0.914 & 0.916 & 0.882 & 0.897 & 0.883 & 0.887 & 0.883 & 0.882 & 1.425 & 1.320 & 1.137 & 1.026 & 0.861 & 0.818 & 0.891 & 0.827 & $50 / 10$ \\
\hline $50 / 10$ & 0.921 & 0.918 & 0.901 & 0.905 & 0.887 & 0.886 & 0.885 & 0.886 & 1.437 & 1.356 & 1.133 & 1.074 & 0.865 & 0.825 & 0.880 & 0.822 & $50 / 10$ \\
\hline $50 / 10$ & 0.919 & 0.892 & 0.893 & 0.884 & 0.896 & 0.889 & 0.881 & 0.873 & 1.424 & 1.288 & 1.116 & 1.042 & 0.858 & 0.812 & 0.896 & 0.827 & $50 / 10$ \\
\hline $50 / 30$ & 0.978 & 0.977 & 0.912 & 0.925 & 0.919 & 0.920 & 0.909 & 0.886 & 1.148 & 1.012 & 0.957 & 0.866 & 0.806 & 0.777 & 0.767 & 0.705 & - \\
\hline $50 / 30$ & 0.981 & 0.934 & 0.897 & 0.878 & 0.894 & 0.885 & 0.862 & 0.853 & 1.069 & 1.023 & 0.939 & 0.877 & 0.754 & 0.740 & 0.728 & 0.680 & - \\
\hline $50 / 30$ & 0.952 & 0.957 & 0.865 & 0.851 & 0.885 & 0.865 & 0.855 & 0.862 & 1.045 & 0.954 & 0.931 & 0.860 & 0.748 & 0.730 & 0.734 & 0.650 & - \\
\hline $50 / 30$ & 0.964 & 0.936 & 0.862 & 0.870 & 0.893 & 0.903 & 0.872 & 0.859 & 1.032 & 0.987 & 0.919 & 0.871 & 0.751 & 0.761 & 0.728 & 0.674 & - \\
\hline $50 / 30$ & 0.935 & 0.967 & 0.913 & 0.886 & 0.894 & 0.888 & 0.883 & 0.874 & 1.036 & 0.957 & 0.947 & 0.859 & 0.759 & 0.745 & 0.727 & 0.653 & - \\
\hline $50 / 30$ & 0.970 & 0.953 & 0.885 & 0.879 & 0.888 & 0.899 & 0.871 & 0.867 & 0.932 & 0.920 & 0.899 & 0.834 & 0.754 & 0.744 & 0.720 & 0.682 & - \\
\hline $50 / 30$ & 0.945 & 0.951 & 0.905 & 0.896 & 0.894 & 0.881 & 0.878 & 0.877 & 1.044 & 0.970 & 0.898 & 0.843 & 0.754 & 0.747 & 0.742 & 0.685 & - \\
\hline $50 / 30$ & 0.966 & 0.949 & 0.900 & 0.880 & 0.883 & 0.900 & 0.887 & 0.883 & 1.075 & 0.960 & 0.924 & 0.838 & 0.753 & 0.765 & 0.735 & 0.683 & $50 / 30$ \\
\hline $50 / 30$ & 0.953 & 0.952 & 0.894 & 0.880 & 0.894 & 0.885 & 0.870 & 0.872 & 1.049 & 0.966 & 0.948 & 0.848 & 0.773 & 0.760 & 0.747 & 0.683 & $50 / 30$ \\
\hline $50 / 30$ & 0.971 & 0.963 & 0.883 & 0.905 & 0.893 & 0.900 & 0.887 & 0.868 & 1.044 & 0.996 & 0.952 & 0.862 & 0.770 & 0.744 & 0.769 & 0.702 & $50 / 30$ \\
\hline $50 / 30$ & 0.958 & 0.963 & 0.879 & 0.882 & 0.876 & 0.897 & 0.862 & 0.854 & 1.033 & 0.964 & 0.917 & 0.872 & 0.766 & 0.751 & 0.725 & 0.681 & $50 / 30$ \\
\hline
\end{tabular}


Table S5. Data set matrix of the fluorescence intensity ratios before and after addition of varying concentrations of ThrH and AThr $\left(I / I_{0}\right)$ generated from the DNA array.

\begin{tabular}{|c|c|c|c|c|c|c|c|c|c|c|c|c|c|c|c|c|}
\hline \multirow{3}{*}{ Analytes } & \multicolumn{8}{|c|}{ pH 7.4} & \multicolumn{8}{|c|}{ pH 5.4} \\
\hline & \multicolumn{2}{|c|}{ DNA1 } & \multicolumn{2}{|c|}{ DNA2 } & \multicolumn{2}{|c|}{ DNA3 } & \multicolumn{2}{|c|}{ DNA5 } & \multicolumn{2}{|c|}{ DNA1 } & \multicolumn{2}{|c|}{ DNA2 } & \multicolumn{2}{|c|}{ DNA3 } & \multicolumn{2}{|c|}{ DNA5 } \\
\hline & Ch1 & $\mathrm{Ch} 2$ & Ch1 & $\mathrm{Ch} 2$ & Ch1 & $\mathrm{Ch} 2$ & Ch1 & Ch2 & Ch1 & $\mathrm{Ch} 2$ & Ch1 & $\mathrm{Ch} 2$ & Ch1 & $\mathrm{Ch} 2$ & Ch1 & Ch2 \\
\hline $0 / 0$ & 1.029 & 1.036 & 1.033 & 1.028 & 1.030 & 1.025 & 1.014 & 1.023 & 1.023 & 1.021 & 1.022 & 1.005 & 1.020 & 1.021 & 0.998 & 0.982 \\
\hline $0 / 0$ & 1.045 & 1.039 & 1.019 & 1.020 & 1.032 & 1.028 & 1.029 & 1.023 & 1.026 & 0.999 & 0.999 & 0.991 & 1.020 & 0.995 & 1.018 & 1.016 \\
\hline $0 / 0$ & 1.034 & 1.031 & 1.031 & 1.003 & 1.011 & 1.015 & 1.024 & 1.026 & 1.019 & 1.023 & 0.984 & 0.983 & 0.999 & 1.001 & 1.003 & 1.007 \\
\hline $0 / 0$ & 1.051 & 1.050 & 1.018 & 1.028 & 1.037 & 1.037 & 1.027 & 1.037 & 1.032 & 1.024 & 1.013 & 1.016 & 1.020 & 1.007 & 1.001 & 0.997 \\
\hline $0 / 0$ & 1.054 & 1.047 & 1.039 & 1.030 & 1.019 & 1.015 & 1.028 & 1.030 & 1.028 & 1.029 & 0.999 & 0.986 & 0.997 & 0.990 & 1.009 & 0.995 \\
\hline $0 / 0$ & 1.029 & 1.043 & 1.016 & 1.006 & 1.027 & 1.025 & 1.034 & 1.028 & 1.014 & 1.015 & 1.019 & 1.007 & 1.031 & 1.018 & 1.004 & 0.992 \\
\hline $0 / 40$ & 1.095 & 1.186 & 0.988 & 1.001 & 0.999 & 0.998 & 1.109 & 1.135 & 0.910 & 0.984 & 0.955 & 0.954 & 0.954 & 0.956 & 0.985 & 0.987 \\
\hline $0 / 40$ & 1.088 & 1.178 & 0.992 & 1.009 & 1.017 & 1.018 & 1.104 & 1.138 & 0.916 & 1.005 & 0.951 & 0.959 & 0.953 & 0.973 & 0.957 & 0.961 \\
\hline $0 / 40$ & 1.082 & 1.176 & 0.997 & 1.007 & 0.988 & 0.993 & 1.077 & 1.102 & 0.930 & 0.991 & 0.937 & 0.936 & 0.935 & 0.933 & 0.975 & 0.971 \\
\hline $0 / 40$ & 1.070 & 1.172 & 0.998 & 0.994 & 1.012 & 1.008 & 1.076 & 1.101 & 0.902 & 0.963 & 0.967 & 0.962 & 0.973 & 0.971 & 0.967 & 0.968 \\
\hline $0 / 40$ & 1.075 & 1.179 & 0.997 & 0.995 & 0.979 & 0.980 & 1.095 & 1.118 & 0.908 & 0.984 & 0.961 & 0.970 & 0.946 & 0.963 & 0.976 & 0.980 \\
\hline $0 / 40$ & 1.077 & 1.162 & 0.984 & 0.991 & 0.996 & 0.989 & 1.079 & 1.116 & 0.920 & 1.000 & 0.967 & 0.978 & 0.937 & 0.951 & 0.957 & 0.966 \\
\hline $0 / 80$ & 1.093 & 1.201 & 0.976 & 0.971 & 1.021 & 1.015 & 1.089 & 1.118 & 0.937 & 1.025 & 0.963 & 0.966 & 0.957 & 0.983 & 0.969 & 0.966 \\
\hline $0 / 80$ & 1.097 & 1.202 & 0.966 & 0.992 & 1.005 & 1.014 & 1.070 & 1.105 & 0.909 & 1.004 & 0.936 & 0.930 & 0.940 & 0.941 & 0.945 & 0.941 \\
\hline $0 / 80$ & 1.120 & 1.232 & 0.993 & 0.994 & 1.008 & 1.016 & 1.099 & 1.121 & 0.926 & 1.041 & 0.946 & 0.959 & 0.969 & 0.981 & 0.954 & 0.955 \\
\hline $0 / 80$ & 1.096 & 1.207 & 0.991 & 1.003 & 0.995 & 1.005 & 1.103 & 1.133 & 0.948 & 1.046 & 0.941 & 0.943 & 0.931 & 0.945 & 0.953 & 0.952 \\
\hline $0 / 80$ & 1.114 & 1.238 & 0.987 & 0.992 & 0.998 & 1.002 & 1.095 & 1.128 & 0.922 & 1.034 & 0.964 & 0.967 & 0.935 & 0.943 & 0.969 & 0.971 \\
\hline $0 / 80$ & 1.077 & 1.191 & 0.966 & 0.965 & 0.988 & 0.992 & 1.080 & 1.105 & 0.925 & 1.019 & 0.957 & 0.951 & 0.935 & 0.939 & 0.945 & 0.947 \\
\hline $0 / 120$ & 1.114 & 1.221 & 0.978 & 0.968 & 0.996 & 0.991 & 1.089 & 1.109 & 0.939 & 1.045 & 0.936 & 0.938 & 0.936 & 0.946 & 0.955 & 0.955 \\
\hline $0 / 120$ & 1.095 & 1.209 & 0.958 & 0.966 & 0.992 & 0.996 & 1.083 & 1.111 & 0.952 & 1.056 & 0.928 & 0.924 & 0.965 & 0.964 & 0.929 & 0.945 \\
\hline $0 / 120$ & 1.098 & 1.220 & 0.965 & 0.971 & 0.975 & 0.986 & 1.043 & 1.069 & 0.917 & 1.015 & 0.923 & 0.941 & 0.929 & 0.943 & 0.955 & 0.966 \\
\hline $0 / 120$ & 1.126 & 1.238 & 0.962 & 0.968 & 0.989 & 1.009 & 1.080 & 1.118 & 0.943 & 1.032 & 0.933 & 0.941 & 0.926 & 0.928 & 0.934 & 0.941 \\
\hline $0 / 120$ & 1.106 & 1.219 & 0.965 & 0.961 & 0.995 & 0.991 & 1.045 & 1.079 & 0.929 & 1.032 & 0.947 & 0.945 & 0.930 & 0.939 & 0.964 & 0.970 \\
\hline $0 / 120$ & 1.096 & 1.204 & 0.970 & 0.979 & 1.001 & 0.996 & 1.072 & 1.099 & 0.928 & 1.024 & 0.945 & 0.939 & 0.952 & 0.966 & 0.965 & 0.973 \\
\hline $100 / 0$ & 1.020 & 1.038 & 1.000 & 0.997 & 1.000 & 0.993 & 1.002 & 1.014 & 0.955 & 0.952 & 0.956 & 0.957 & 0.967 & 0.976 & 0.968 & 0.964 \\
\hline $100 / 0$ & 1.002 & 1.017 & 0.999 & 0.991 & 0.988 & 0.993 & 1.015 & 1.018 & 0.998 & 1.002 & 0.969 & 0.971 & 0.983 & 0.991 & 0.961 & 0.944 \\
\hline $100 / 0$ & 0.997 & 1.020 & 0.990 & 0.996 & 0.982 & 0.972 & 0.982 & 0.987 & 0.959 & 0.957 & 0.962 & 0.955 & 0.971 & 0.964 & 0.979 & 0.975 \\
\hline $100 / 0$ & 1.014 & 1.026 & 0.993 & 0.994 & 0.973 & 0.980 & 1.001 & 1.001 & 0.966 & 0.975 & 0.974 & 0.971 & 0.976 & 0.980 & 0.978 & 0.970 \\
\hline $100 / 0$ & 1.007 & 1.028 & 0.990 & 0.995 & 0.992 & 0.979 & 0.984 & 0.994 & 0.975 & 0.968 & 0.985 & 0.990 & 0.978 & 0.987 & 0.987 & 0.969 \\
\hline $100 / 0$ & 1.004 & 0.996 & 0.986 & 0.967 & 0.975 & 0.978 & 0.996 & 1.000 & 0.953 & 0.954 & 0.961 & 0.928 & 0.946 & 0.948 & 0.963 & 0.955 \\
\hline $100 / 40$ & 1.022 & 1.054 & 0.993 & 1.000 & 0.994 & 0.993 & 1.031 & 1.029 & 0.861 & 0.918 & 0.974 & 0.963 & 0.987 & 0.994 & 0.974 & 0.974 \\
\hline $100 / 40$ & 1.040 & 1.047 & 0.987 & 0.990 & 0.997 & 0.987 & 1.036 & 1.042 & 0.869 & 0.916 & 0.987 & 0.970 & 1.000 & 1.004 & 0.981 & 0.981 \\
\hline $100 / 40$ & 1.037 & 1.059 & 0.985 & 0.986 & 0.974 & 0.975 & 0.993 & 0.993 & 0.867 & 0.926 & 0.952 & 0.978 & 0.957 & 0.972 & 0.967 & 0.955 \\
\hline $100 / 40$ & 1.032 & 1.054 & 1.023 & 1.007 & 1.008 & 1.010 & 1.008 & 1.017 & 0.865 & 0.914 & 0.945 & 0.949 & 0.960 & 0.972 & 0.961 & 0.956 \\
\hline $100 / 40$ & 1.024 & 1.033 & 0.988 & 0.978 & 1.007 & 1.000 & 1.021 & 1.018 & 0.857 & 0.911 & 0.928 & 0.928 & 0.927 & 0.926 & 0.939 & 0.944 \\
\hline $100 / 40$ & 1.022 & 1.031 & 1.002 & 0.994 & 0.979 & 0.987 & 1.009 & 1.017 & 0.857 & 0.911 & 0.946 & 0.953 & 0.967 & 0.980 & 0.968 & 0.977 \\
\hline $100 / 80$ & 1.050 & 1.069 & 0.978 & 0.981 & 0.989 & 0.999 & 1.027 & 1.045 & 0.817 & 0.877 & 0.924 & 0.929 & 0.946 & 0.957 & 0.939 & 0.937 \\
\hline $100 / 80$ & 1.024 & 1.051 & 0.982 & 0.983 & 0.978 & 0.986 & 1.033 & 1.054 & 0.847 & 0.925 & 0.916 & 0.909 & 0.943 & 0.954 & 0.944 & 0.946 \\
\hline $100 / 80$ & 1.019 & 1.063 & 0.981 & 0.995 & 0.979 & 0.978 & 1.028 & 1.045 & 0.821 & 0.884 & 0.929 & 0.940 & 0.950 & 0.959 & 0.934 & 0.944 \\
\hline $100 / 80$ & 1.030 & 1.068 & 0.977 & 0.979 & 0.983 & 0.986 & 1.043 & 1.052 & 0.837 & 0.896 & 0.915 & 0.902 & 0.956 & 0.965 & 0.938 & 0.939 \\
\hline $100 / 80$ & 1.027 & 1.058 & 0.977 & 0.978 & 0.986 & 0.982 & 1.028 & 1.045 & 0.831 & 0.894 & 0.931 & 0.935 & 0.954 & 0.961 & 0.938 & 0.936 \\
\hline $100 / 80$ & 1.019 & 1.051 & 1.006 & 1.000 & 0.984 & 0.987 & 1.025 & 1.037 & 0.822 & 0.893 & 0.926 & 0.931 & 0.943 & 0.961 & 0.937 & 0.939 \\
\hline $100 / 120$ & 1.060 & 1.151 & 1.003 & 1.014 & 1.003 & 0.995 & 1.097 & 1.128 & 0.854 & 0.938 & 0.902 & 0.895 & 0.915 & 0.920 & 0.967 & 0.968 \\
\hline $100 / 120$ & 1.066 & 1.149 & 0.977 & 0.980 & 1.003 & 1.012 & 1.099 & 1.131 & 0.861 & 0.939 & 0.913 & 0.904 & 0.961 & 0.981 & 0.969 & 0.970 \\
\hline $100 / 120$ & 1.055 & 1.171 & 0.969 & 0.976 & 0.989 & 0.990 & 1.065 & 1.095 & 0.906 & 0.984 & 0.903 & 0.901 & 0.922 & 0.923 & 0.963 & 0.965 \\
\hline $100 / 120$ & 1.039 & 1.158 & 0.974 & 0.966 & 0.991 & 0.993 & 1.069 & 1.100 & 0.858 & 0.947 & 0.891 & 0.900 & 0.918 & 0.919 & 0.936 & 0.956 \\
\hline $100 / 120$ & 1.061 & 1.158 & 0.971 & 0.974 & 1.001 & 1.001 & 1.077 & 1.103 & 0.866 & 0.953 & 0.929 & 0.929 & 0.979 & 0.999 & 0.950 & 0.944 \\
\hline $100 / 120$ & 1.085 & 1.176 & 1.007 & 1.003 & 0.972 & 0.971 & 1.114 & 1.131 & 0.883 & 0.972 & 0.911 & 0.905 & 0.948 & 0.953 & 0.980 & 0.974 \\
\hline
\end{tabular}


Table S6. Effect of selected TAMRA-modified ssDNAs on the classification accuracy of the DNA array for the detection of complexes between ThrH and AThr in the presence of human serum.

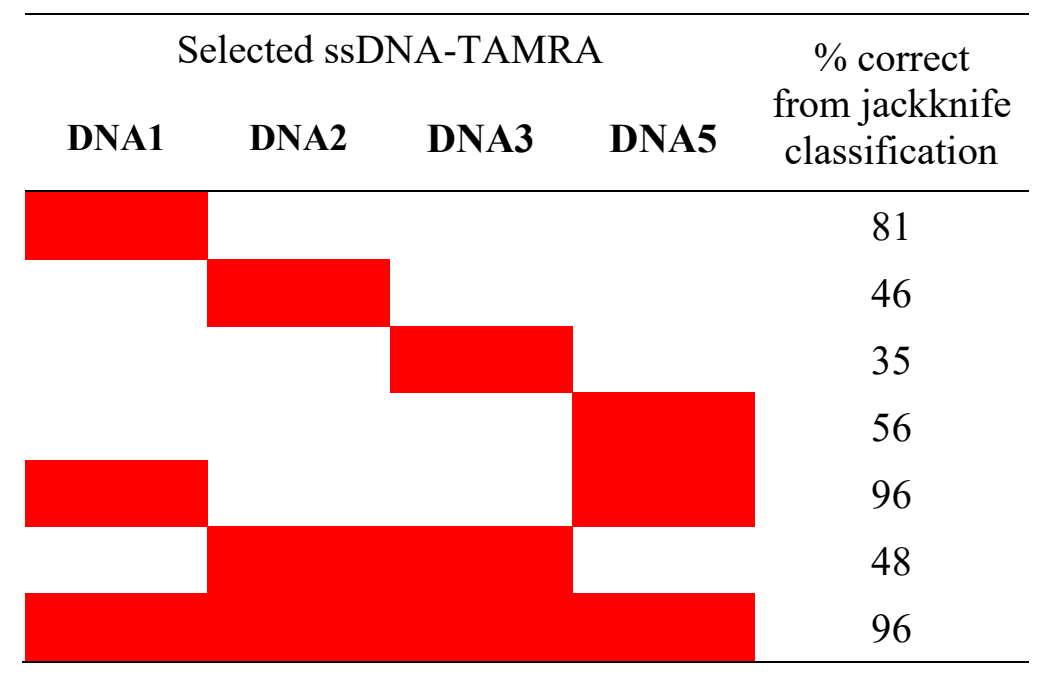

Table S7. Complementary DNA molecules used in this study. In order to avoid changes in the fluorescence of the corresponding ssDNA-TAMRAs due to hybridization, one nucleobase at the 5'terminus was removed.

\begin{tabular}{lcc}
\hline DNA sequence & code & corresponding ssDNA-TAMRA \\
\hline 5'-CA ACC ACA CCA ACC-3' & cDNA1 & DNA1 \\
5'-CGA CGC CCA CCA TGC CGT-3' & cDNA2 & DNA2 \\
\hline
\end{tabular}


Table S8. Data set matrix of the fluorescence intensity ratios before and after addition of $50 \mu \mathrm{g} / \mathrm{mL}$ proteases to solutions containing ssDNA-TAMRAs $\left(I_{\text {without }} / I_{0}\right)$ and the intensity ratios when both proteases and the corresponding complementary ssDNAs were added against $I_{0}\left(I_{\text {with }} / I_{0}\right)$ generated from the DNA array.

\begin{tabular}{|c|c|c|c|c|c|c|c|c|}
\hline & \multicolumn{4}{|c|}{ DNA1 $/ \mathrm{pH}=7.4$} & \multicolumn{4}{|c|}{ DNA2 $/ \mathrm{pH}=7.4$} \\
\hline & \multicolumn{2}{|c|}{ without cDNA1 $\left(I_{\text {without }} / I_{0}\right)$} & \multicolumn{2}{|c|}{ with cDNA1 $\left(I_{\text {with }} / I_{0}\right)$} & \multicolumn{2}{|c|}{ without cDNA2 $\left(I_{\text {without }} / I_{0}\right)$} & \multicolumn{2}{|c|}{ with cDNA2 $\left(I_{\text {with }} / I_{0}\right)$} \\
\hline & Ch1 & $\mathrm{Ch} 2$ & Ch1 & $\mathrm{Ch} 2$ & Ch1 & $\mathrm{Ch} 2$ & Ch1 & $\mathrm{Ch} 2$ \\
\hline Car & 1.069 & 1.061 & 0.994 & 1.004 & 1.000 & 0.998 & 0.901 & 0.875 \\
\hline Car & 1.052 & 1.057 & 0.977 & 1.013 & 0.995 & 1.001 & 0.894 & 0.898 \\
\hline Car & 1.065 & 1.069 & 1.003 & 1.022 & 0.997 & 0.991 & 0.898 & 0.889 \\
\hline Car & 1.067 & 1.073 & 1.000 & 1.021 & 1.006 & 0.993 & 0.908 & 0.899 \\
\hline Car & 1.088 & 1.057 & 1.028 & 1.025 & 1.008 & 0.994 & 0.901 & 0.890 \\
\hline Car & 1.073 & 1.059 & 1.008 & 1.028 & 1.005 & 1.000 & 0.915 & 0.907 \\
\hline Chy & 1.084 & 1.056 & 0.992 & 1.002 & 0.911 & 0.882 & 0.601 & 0.585 \\
\hline Chy & 1.080 & 1.043 & 0.983 & 1.003 & 0.912 & 0.875 & 0.596 & 0.584 \\
\hline Chy & 1.093 & 1.068 & 1.002 & 1.015 & 0.922 & 0.883 & 0.600 & 0.586 \\
\hline Chy & 1.085 & 1.051 & 1.000 & 0.990 & 0.904 & 0.879 & 0.585 & 0.591 \\
\hline Chy & 1.083 & 1.071 & 1.001 & 1.009 & 0.923 & 0.898 & 0.615 & 0.599 \\
\hline Chy & 1.098 & 1.055 & 1.004 & 1.004 & 0.918 & 0.904 & 0.607 & 0.605 \\
\hline Ela & 0.961 & 0.948 & 0.914 & 0.903 & 0.921 & 0.912 & 0.844 & 0.834 \\
\hline Ela & 0.944 & 0.922 & 0.899 & 0.893 & 0.924 & 0.929 & 0.861 & 0.851 \\
\hline Ela & 0.950 & 0.957 & 0.895 & 0.904 & 0.916 & 0.910 & 0.847 & 0.851 \\
\hline Ela & 0.954 & 0.949 & 0.911 & 0.913 & 0.923 & 0.915 & 0.849 & 0.837 \\
\hline Ela & 0.963 & 0.970 & 0.913 & 0.927 & 0.927 & 0.936 & 0.861 & 0.856 \\
\hline Ela & 0.957 & 0.952 & 0.910 & 0.917 & 0.933 & 0.919 & 0.858 & 0.859 \\
\hline Pap & 0.971 & 0.963 & 0.627 & 0.667 & 0.629 & 0.629 & 0.372 & 0.384 \\
\hline Pap & 0.943 & 0.938 & 0.611 & 0.653 & 0.637 & 0.635 & 0.376 & 0.382 \\
\hline Pap & 0.956 & 0.961 & 0.618 & 0.658 & 0.621 & 0.629 & 0.378 & 0.388 \\
\hline Pap & 0.938 & 0.935 & 0.611 & 0.662 & 0.634 & 0.636 & 0.374 & 0.386 \\
\hline Pap & 0.964 & 0.965 & 0.623 & 0.665 & 0.648 & 0.637 & 0.379 & 0.385 \\
\hline Pap & 0.959 & 0.931 & 0.611 & 0.647 & 0.646 & 0.658 & 0.382 & 0.399 \\
\hline Pro & 1.012 & 1.010 & 0.947 & 0.979 & 0.957 & 0.960 & 0.879 & 0.885 \\
\hline Pro & 1.003 & 0.991 & 0.933 & 0.939 & 0.974 & 0.967 & 0.906 & 0.896 \\
\hline Pro & 1.018 & 1.001 & 0.963 & 0.975 & 0.982 & 0.973 & 0.905 & 0.892 \\
\hline Pro & 0.996 & 0.988 & 0.939 & 0.963 & 0.983 & 0.979 & 0.909 & 0.910 \\
\hline Pro & 1.021 & 1.006 & 0.978 & 0.992 & 0.967 & 0.972 & 0.890 & 0.900 \\
\hline Pro & 0.994 & 1.007 & 0.939 & 0.964 & 0.979 & 0.968 & 0.912 & 0.901 \\
\hline Thr & 1.165 & 1.293 & 1.024 & 1.069 & 0.995 & 0.994 & 0.621 & 0.624 \\
\hline Thr & 1.181 & 1.239 & 1.030 & 1.057 & 0.988 & 0.989 & 0.621 & 0.618 \\
\hline Thr & 1.177 & 1.270 & 1.036 & 1.085 & 1.001 & 0.994 & 0.627 & 0.621 \\
\hline Thr & 1.156 & 1.256 & 1.035 & 1.074 & 0.996 & 0.990 & 0.623 & 0.620 \\
\hline Thr & 1.183 & 1.298 & 1.044 & 1.108 & 1.008 & 1.001 & 0.628 & 0.630 \\
\hline Thr & 1.162 & 1.260 & 1.028 & 1.078 & 1.007 & 0.990 & 0.634 & 0.622 \\
\hline TryB & 1.033 & 1.013 & 0.780 & 0.811 & 0.869 & 0.858 & 0.397 & 0.421 \\
\hline TryB & 1.037 & 1.029 & 0.762 & 0.808 & 0.869 & 0.851 & 0.403 & 0.416 \\
\hline TryB & 1.054 & 1.047 & 0.788 & 0.838 & 0.878 & 0.871 & 0.407 & 0.428 \\
\hline TryB & 1.018 & 1.025 & 0.760 & 0.818 & 0.872 & 0.866 & 0.411 & 0.427 \\
\hline TryB & 1.044 & 1.056 & 0.798 & 0.854 & 0.884 & 0.880 & 0.408 & 0.429 \\
\hline TryB & 1.044 & 1.041 & 0.775 & 0.814 & 0.874 & 0.875 & 0.409 & 0.430 \\
\hline TryP & 1.014 & 1.019 & 0.967 & 0.989 & 0.981 & 0.970 & 0.923 & 0.908 \\
\hline TryP & 1.015 & 1.019 & 0.978 & 0.983 & 0.980 & 0.985 & 0.916 & 0.927 \\
\hline TryP & 1.005 & 1.008 & 0.976 & 0.974 & 0.984 & 0.980 & 0.929 & 0.927 \\
\hline TryP & 1.006 & 1.002 & 0.963 & 0.972 & 0.984 & 0.979 & 0.931 & 0.919 \\
\hline TryP & 1.022 & 1.006 & 0.978 & 0.989 & 0.997 & 0.994 & 0.932 & 0.932 \\
\hline TryP & 1.014 & 1.006 & 0.960 & 0.980 & 0.990 & 0.993 & 0.929 & 0.925 \\
\hline
\end{tabular}


(a)

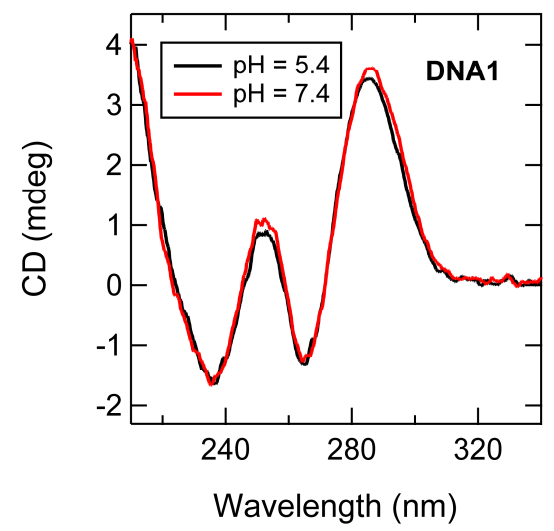

(c)

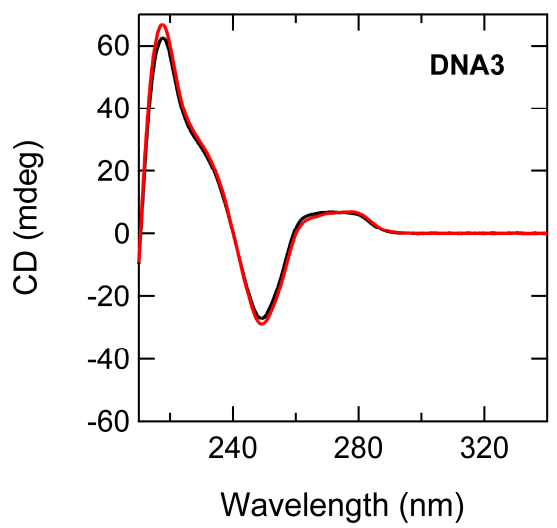

(b)

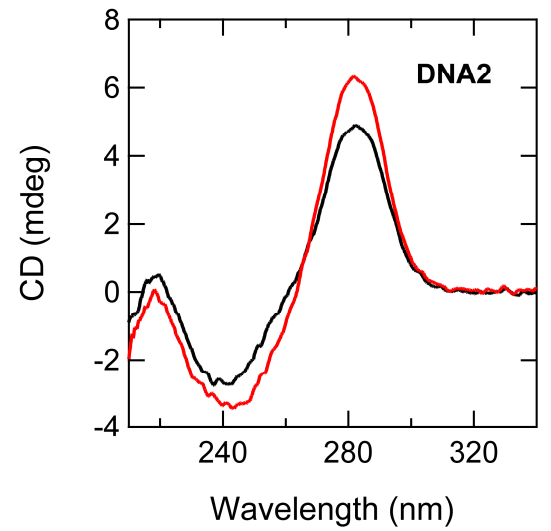

(d)

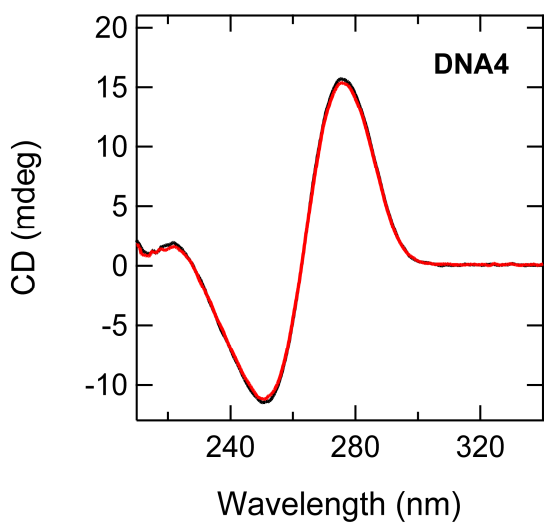

Figure S1. Far-UV CD spectra of ssDNA samples without the fluorophore label at $50 \mu \mathrm{M}$ in $20 \mathrm{mM}$ MOPS ( $\mathrm{pH}=7.4)$ and $20 \mathrm{mM}$ MES $(\mathrm{pH}=5.4)$. Consistent with other reports, DNA1, a typical quadraplexformative sequence, shows a positive maximum at $\sim 285 \mathrm{~nm}$ and a negative maximum at $\sim 265 \mathrm{~nm}^{3}$ A characteristic broad peak ranging from 260 to $280 \mathrm{~nm}$ was observed in the case of oligo(dA) (DNA3) due to a mixture of stacked and unstacked neighboring bases at $25^{\circ} \mathrm{C} .{ }^{4}$ While the spectrum of DNA3 displays a large band at $217 \mathrm{~nm}$, that of oligo(dT) (DNA4) is small in this region. ${ }^{4}$ 


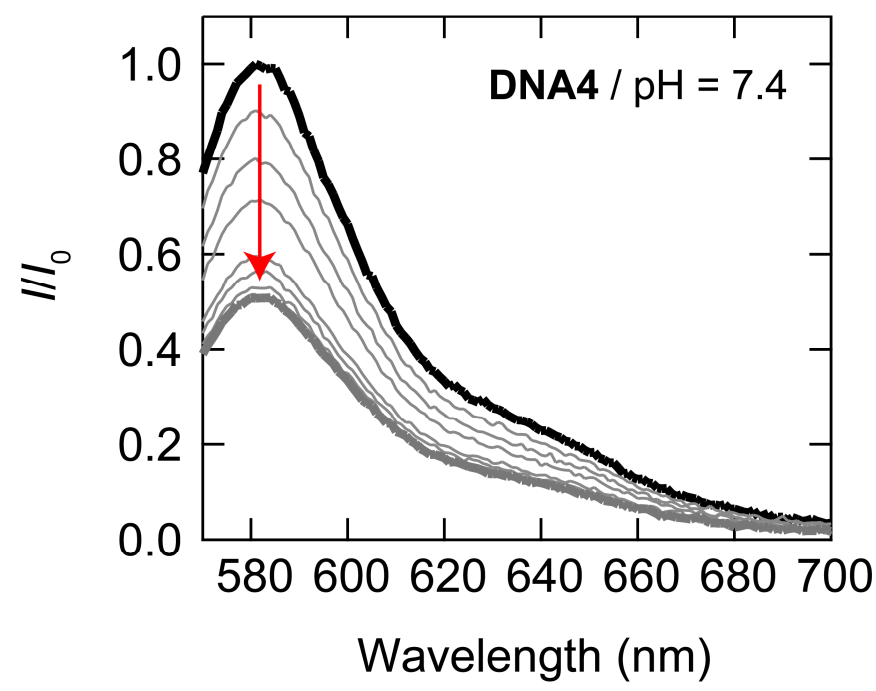

Figure S2. Typical fluorescence spectra of TAMRA-modified ssDNA upon addition of Pap. Pap (0-50 $\mu \mathrm{g} / \mathrm{mL})$ was mixed with DNA4 $(20 \mathrm{nM})$ in $20 \mathrm{mM}$ MOPS $(\mathrm{pH}=7.4) ; \lambda_{\mathrm{ex}}=530 \mathrm{~nm}$.

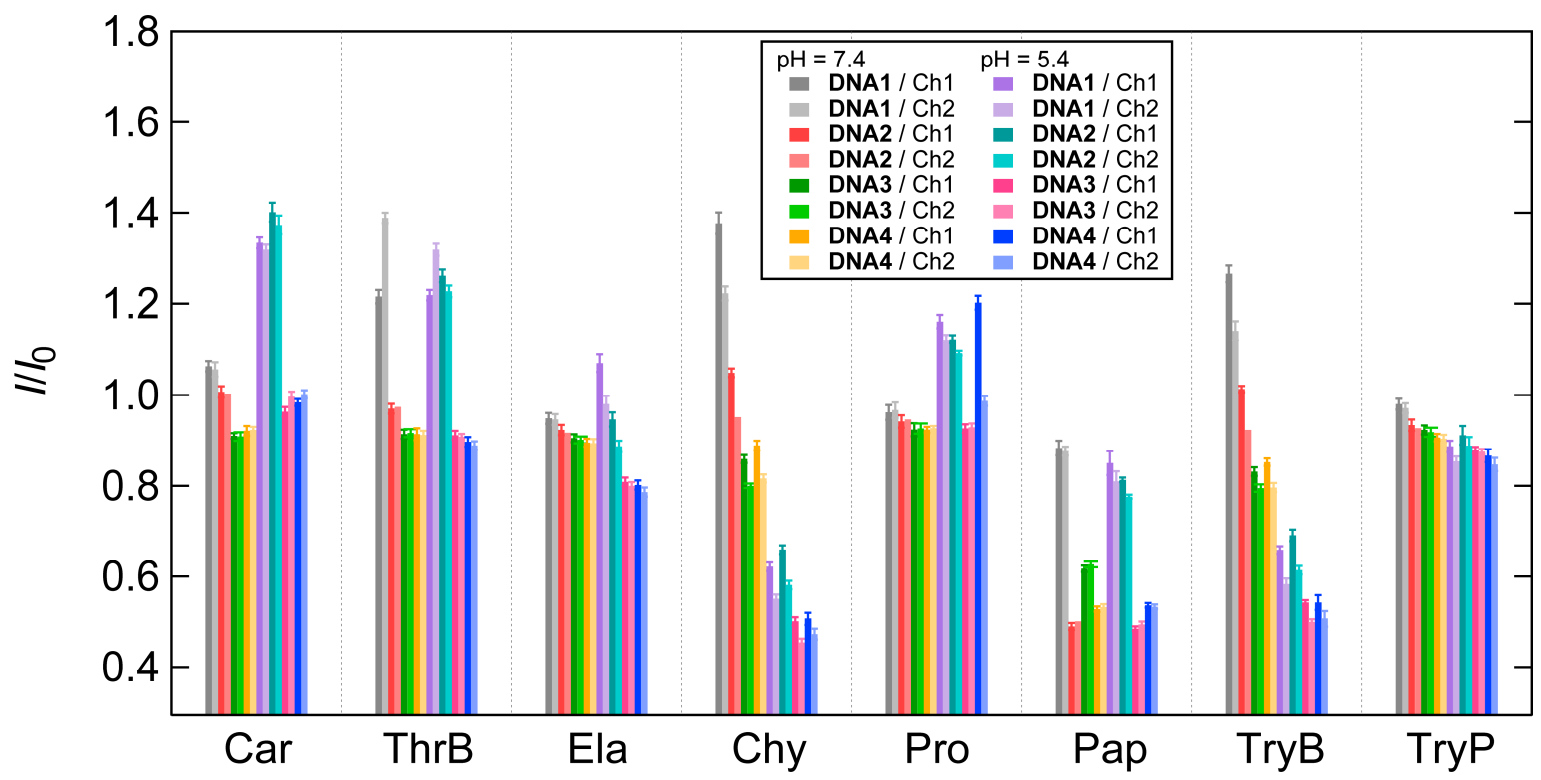

Figure S3. Optical fingerprints of the changes in fluorescence intensity upon addition of $50 \mu \mathrm{g} / \mathrm{mL}$ proteases. Values shown are mean values $\pm 1 \mathrm{SD}(n=11)$. 


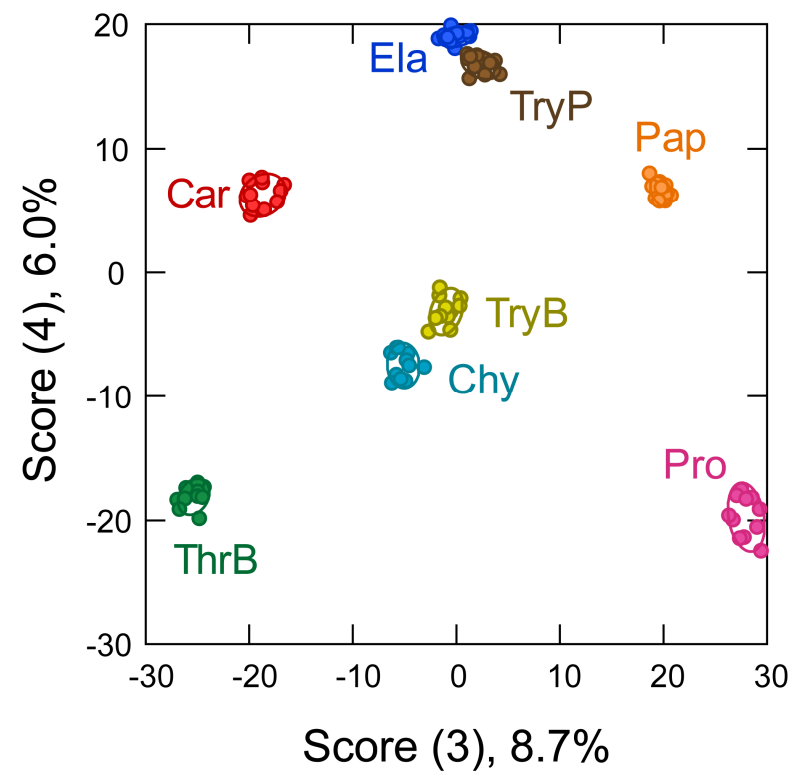

Figure S4. Two-dimensional plot of score (3) vs score (4) for $50 \mu \mathrm{g} / \mathrm{mL}$ proteases obtained from the array of TAMRA-modified ssDNAs, where the ellipsoids represent the confidence intervals $( \pm 1 \mathrm{SD})$ for the individual analytes.

(a)

(b)
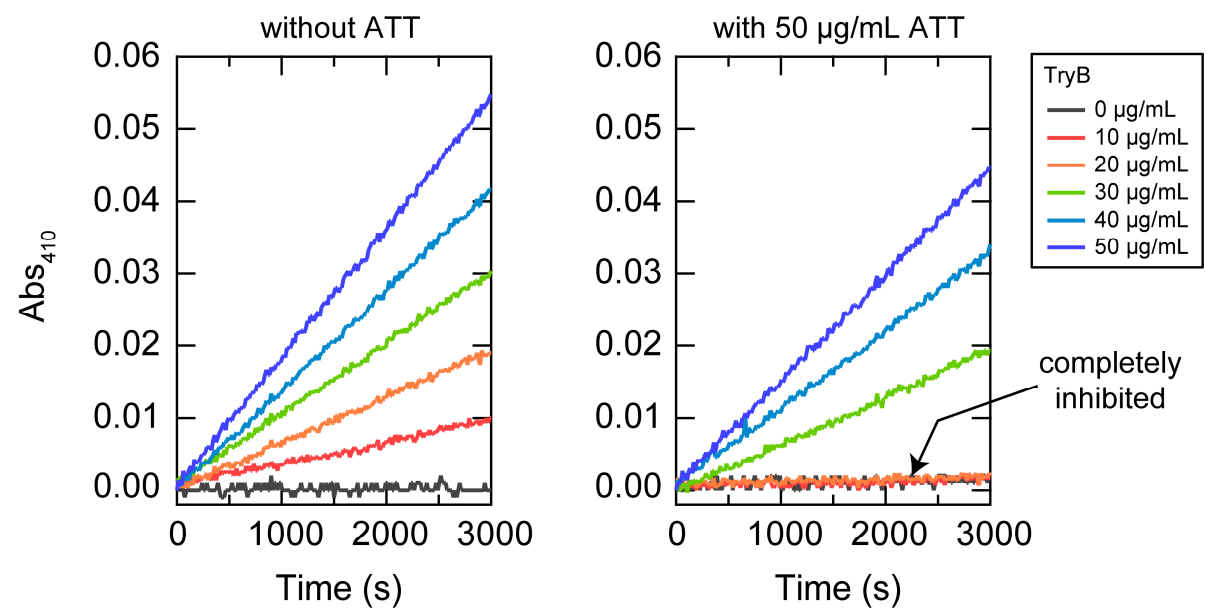

Figure S5. Time course of the increase in absorbance at $410 \mathrm{~nm}$ in the (a) absence and (b) presence of 50 $\mu \mathrm{g} / \mathrm{mL}$ ATT. The solutions contained 0-50 $\mu \mathrm{g} / \mathrm{mL}$ TryB, $2.5 \mathrm{mM}$ L-BAPA, and $20 \mathrm{mM}$ MOPS buffer $(\mathrm{pH}=7.4)$ with/without $50 \mu \mathrm{g} / \mathrm{mL}$ ATT. 


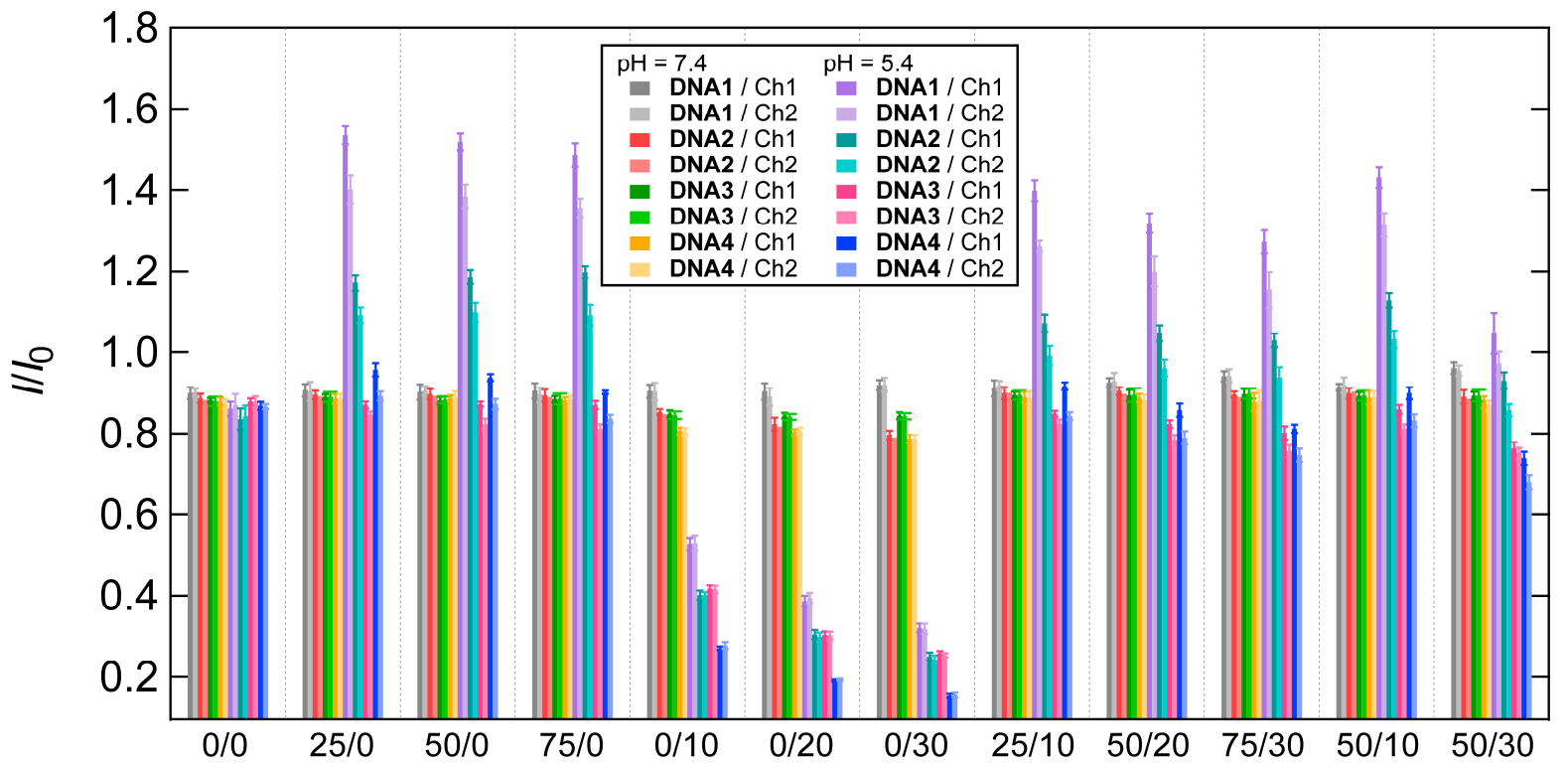

Figure S6. Optical fingerprints of the changes in fluorescence intensity upon addition of mixtures of TryB and its inhibitor ATT. Values shown are mean values $\pm 1 \mathrm{SD}(n=11)$. 
(a)

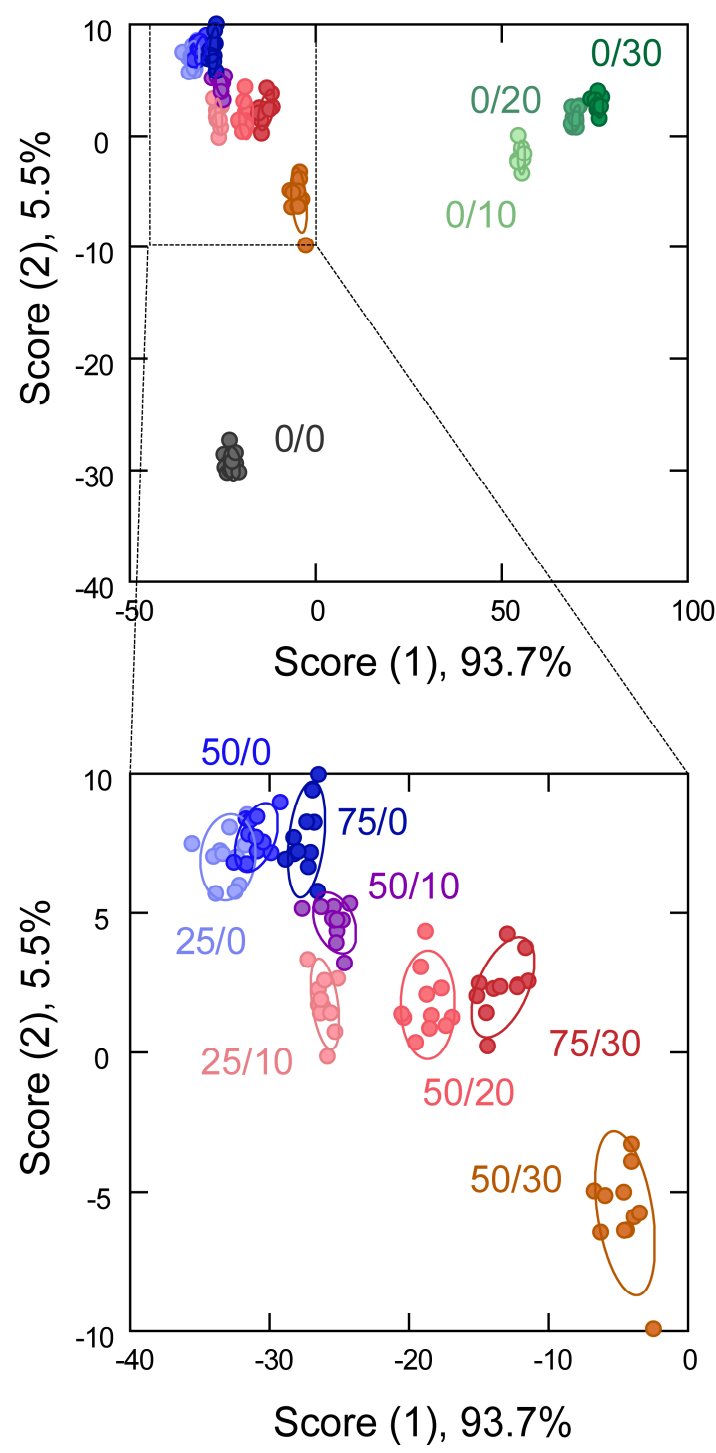

(b)

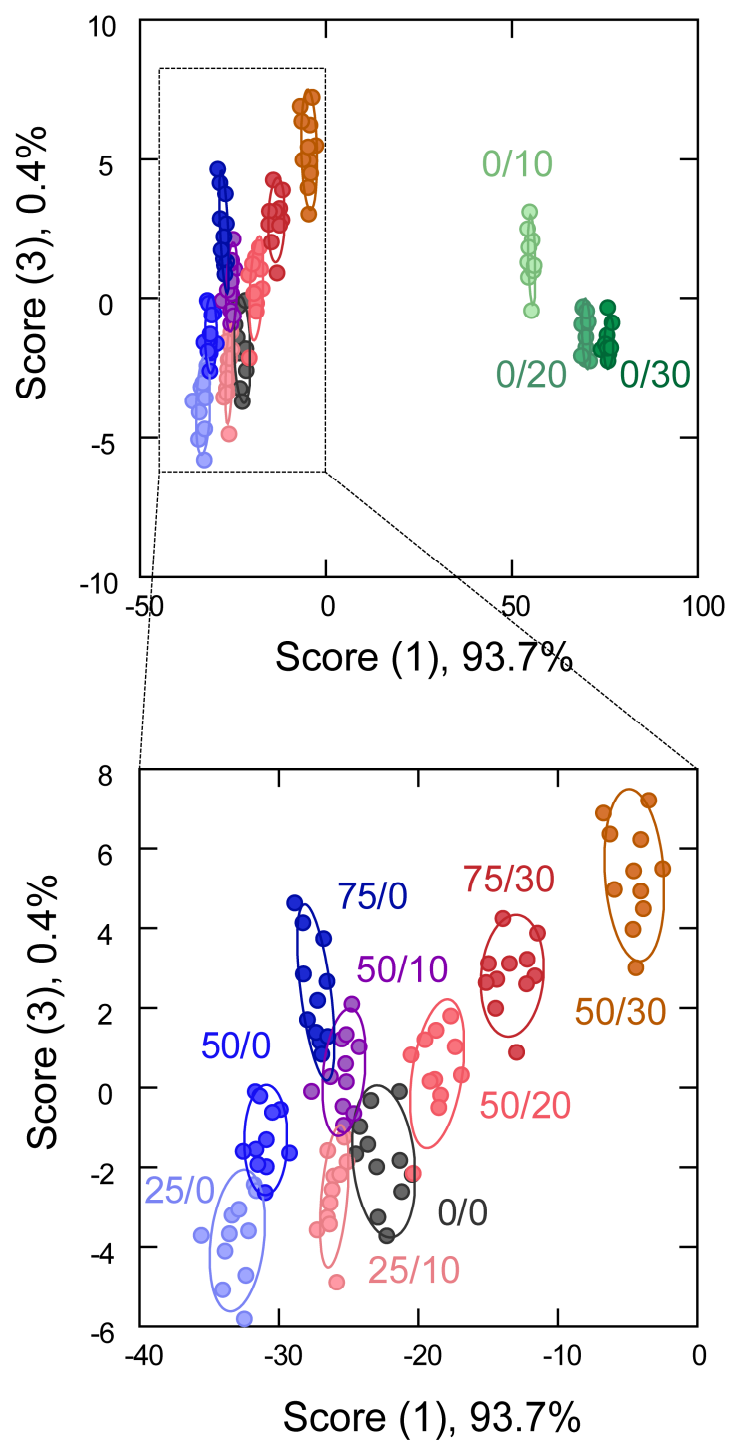

Figure S7. Discriminant score plots for mixtures of TryB and its inhibitor ATT, obtained from the array of TAMRA-modified ssDNAs, where the ellipsoids represent the confidence intervals ( $\pm 1 \mathrm{SD})$ for each analyte. (A) score (1) vs score (2), and (B) score (1) vs score (3). 
(a)

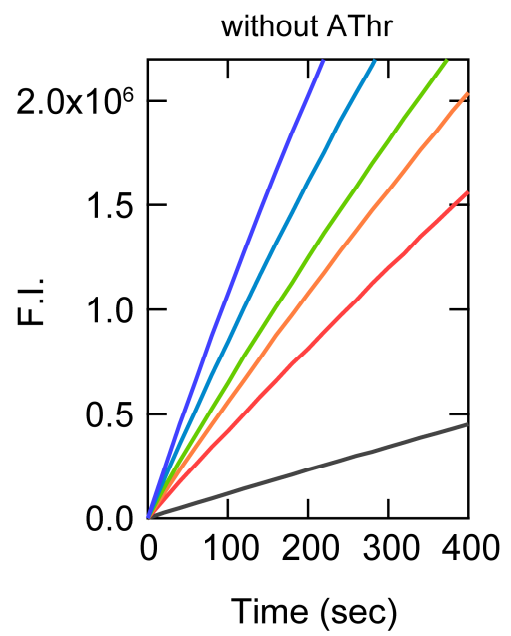

(b)

(c)

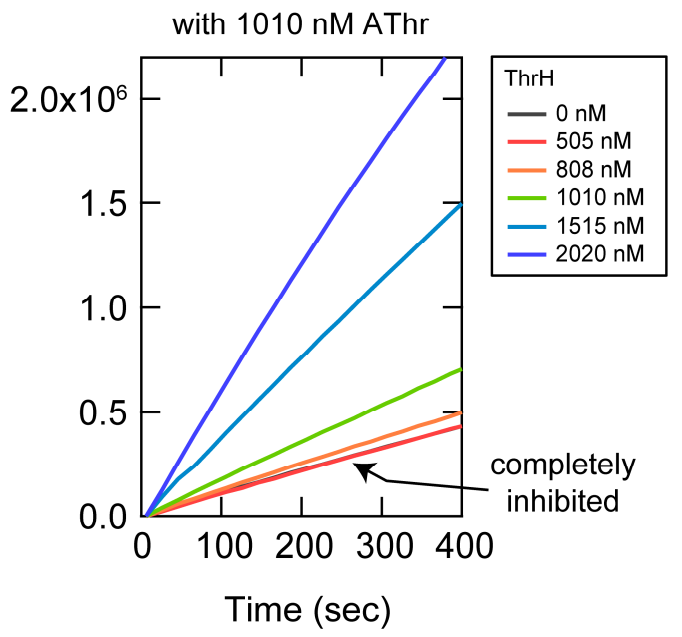

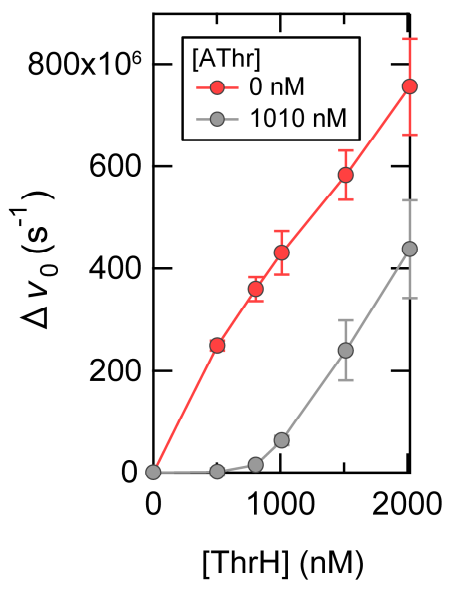

Figure S8. Time course of the increase in fluorescence intensity at $460 \mathrm{~nm}$ in the (a) absence and (b) presence of $1010 \mathrm{nM}$ AThr. The final concentrations for activity determination were $0-2 \mathrm{nM} \mathrm{ThrH,} 0.1$ mM Boc-Val-Pro-Arg-MCA, 1\% human serum, and $30 \mathrm{mM}$ MOPS buffer $(\mathrm{pH}=7.4)$ with/without $1 \mathrm{nM}$ AThr. (c) Changes in the initial rate $\left(\Delta v_{0}\right)$ of the catalytic cleavage of Boc-Val-Pro-Arg-MCA at different concentrations of ThrH in the absence or presence of AThr. Due to the presence of human serum, the substrate was cleaved even without ThrH, hence changes are observed even in the absence of ThrH.

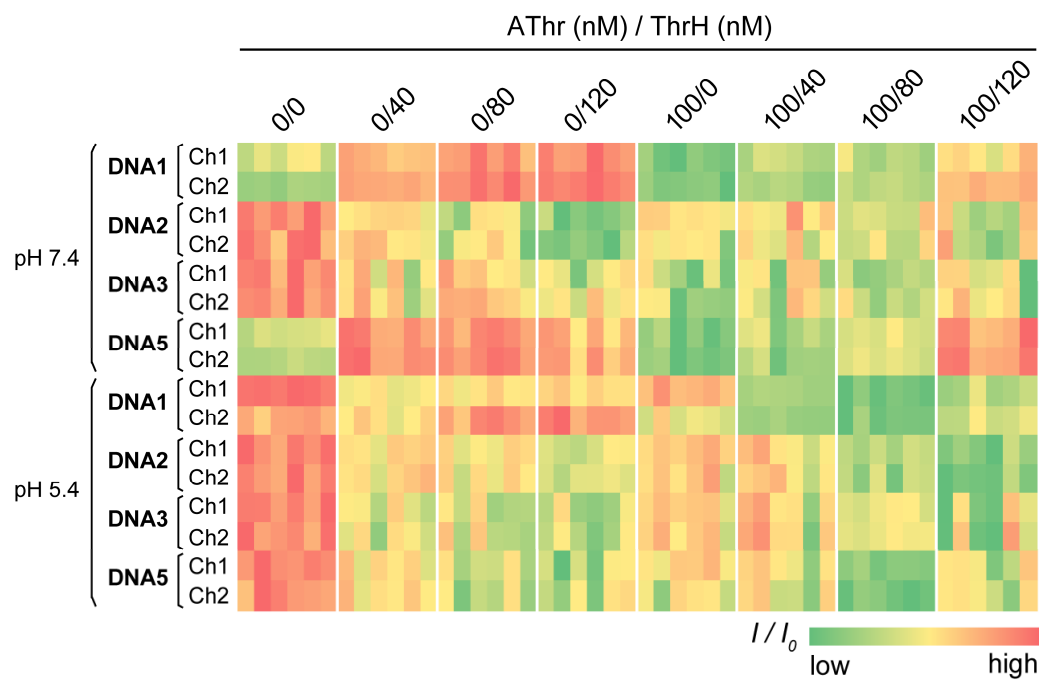

Figure S9. Heat map of the fluorescence fingerprints of various compositions of ThrH and AThr. Six replicates are shown for each analyte. 

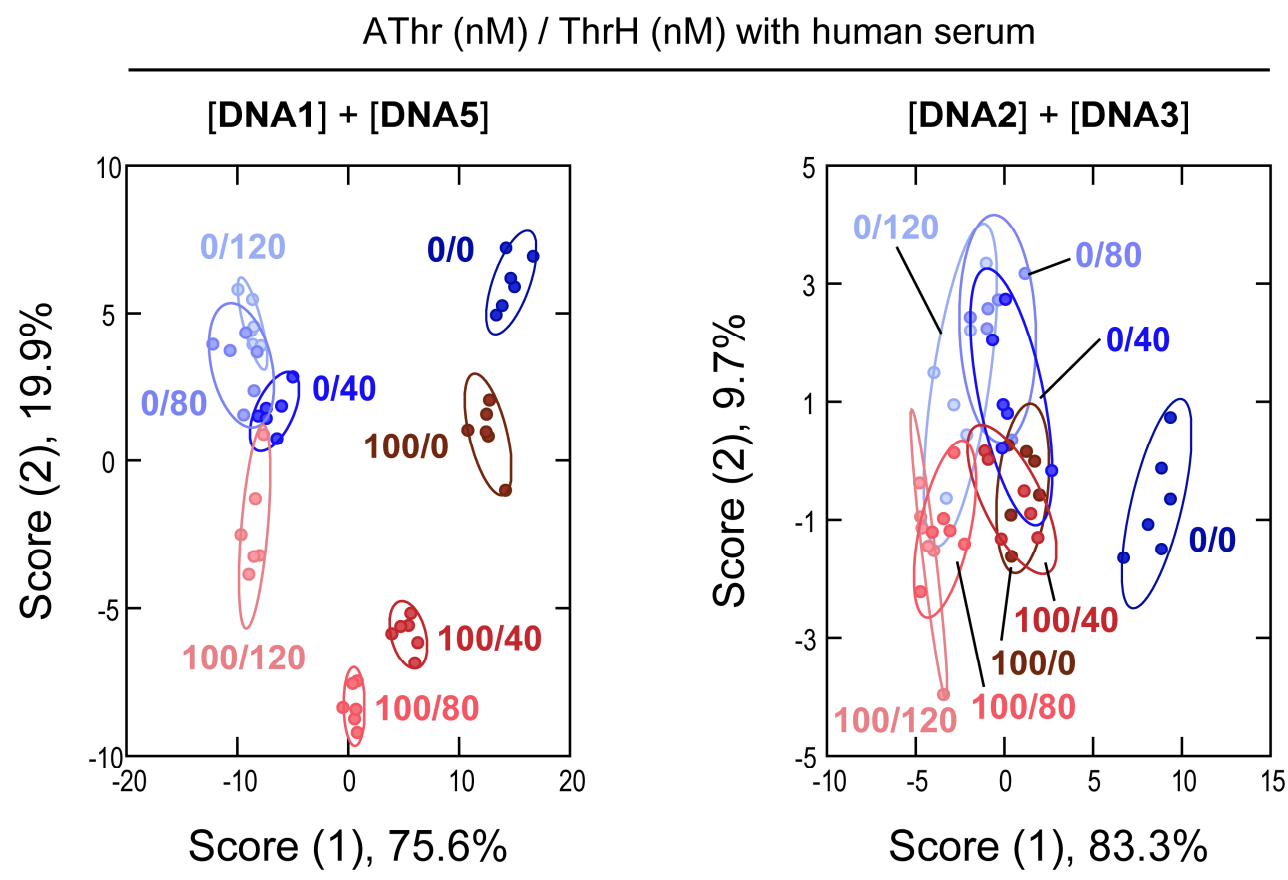

Figure S10. Discriminant score plots for the mixtures containing 0-120 nM ThrH with 1\% human serum in the absence/presence of $100 \mathrm{nM}$ AThr obtained from the array consisting of ThrH aptamers (DNA1 and DNA5) (left) or ssDNAs without specificity to ThrH (DNA2 and DNA3) (right), whereby ellipsoids represent the confidence interval $( \pm 1 \mathrm{SD})$ for each analyte.

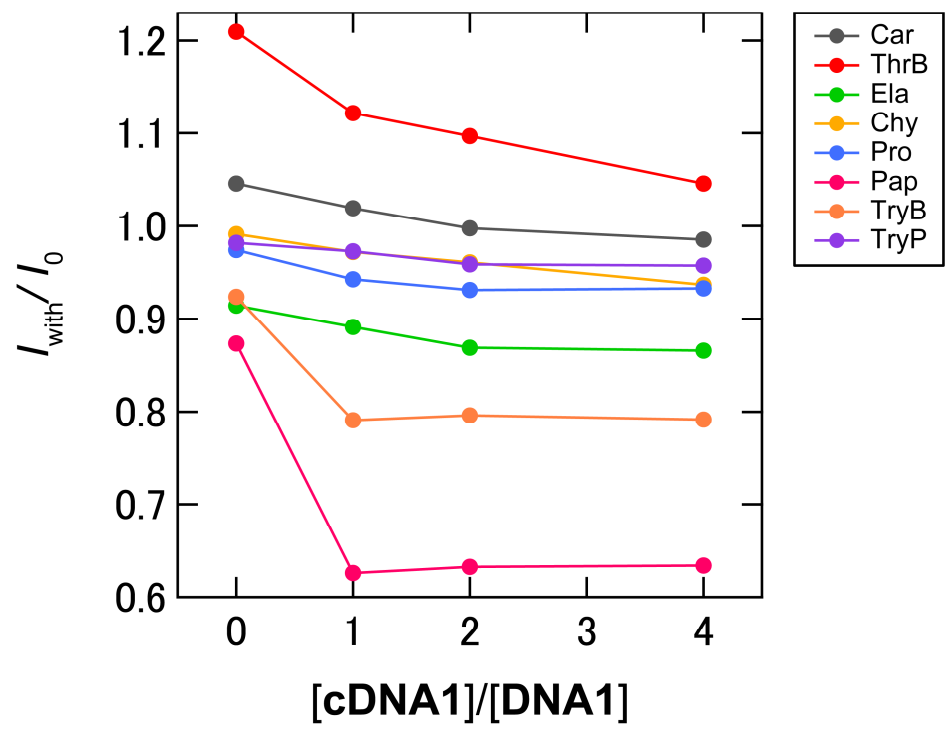

Figure S11. Changes in fluorescence intensity when 0-4 equiv of cDNA1 were added to solutions containing $19.2 \mathrm{nM}$ DNA1, $9.6 \mu \mathrm{g} / \mathrm{mL}$ proteases, and $24 \mathrm{mM}$ MOPS buffer $(\mathrm{pH}=7.0) ; \lambda_{\mathrm{ex}}=525 \mathrm{~nm} ; \lambda_{\mathrm{em}}$ $=595 \mathrm{~nm}$. 
(a)

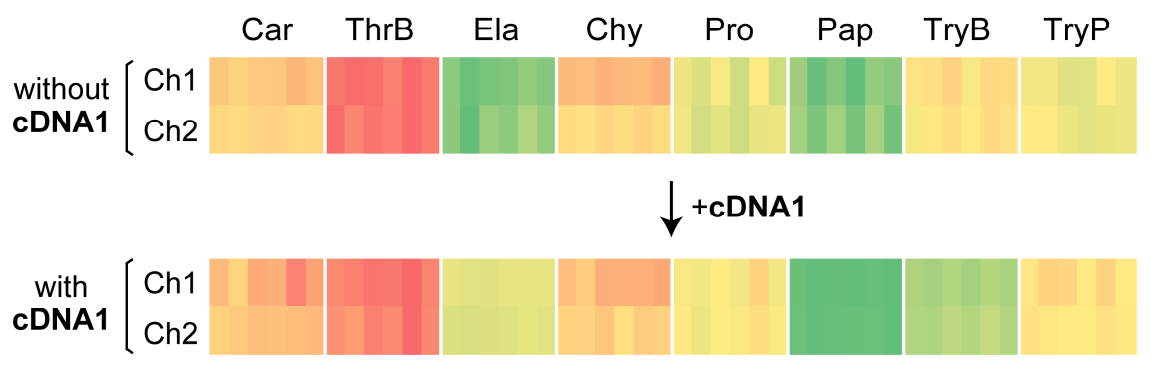

(b)

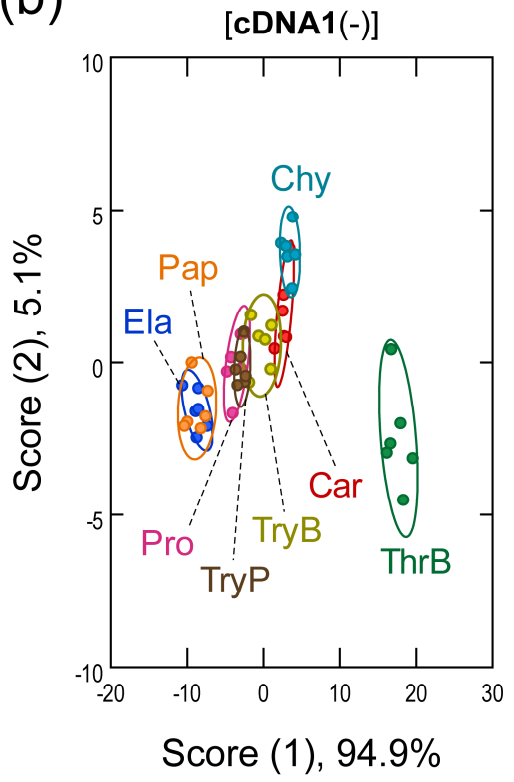

(c)

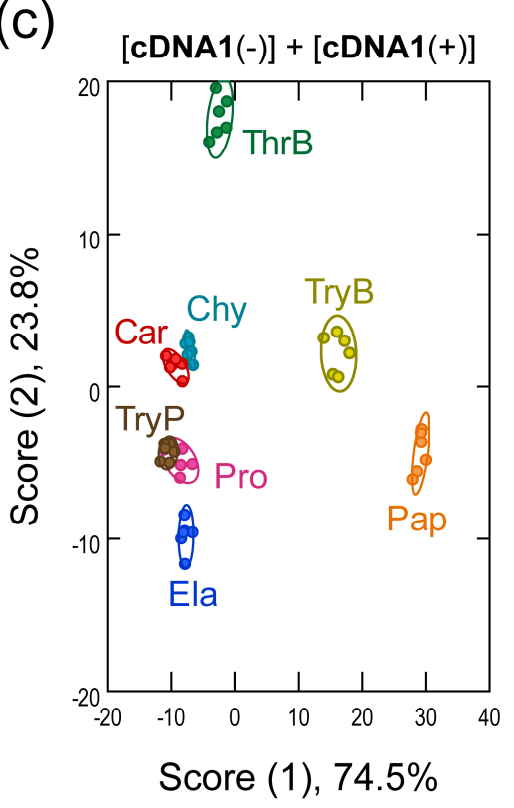

Figure S12. Doubling a dataset of protease responses by hybridization of ssDNA. (a) Heat map of the fluorescence fingerprints of $10 \mu \mathrm{g} / \mathrm{mL}$ proteases with $20 \mathrm{mM}$ MOPS buffer $(\mathrm{pH}=7.4$ ) obtained from DNA1 before/after the addition of cDNA1. Six replicates are shown for each analyte. (b, c) Discriminant score plot for $10 \mu \mathrm{g} / \mathrm{mL}$ proteases for (b) DNA1 only and (c) combined dataset with cDNA1 added, whereby ellipsoids represent the confidence interval ( \pm 1 SD) for each analyte. The accuracy was $54 \%$ based on a jackknife cross-validation test in the absence of cDNA1, while $96 \%$ was achieved when adding the data for cDNA1. 


\section{References}

1. Huntington, J. A.; Thrombin Inhibition by the Serpins. J. Thromb. Haemost., 2013, 11, 254-264.

2. Pace, C. N.; Vajdos, F.; Fee, L.; Grimsley, G.; Gray, T. How to Measure and Predict the Molar Absorption Coefficient of a Protein. Protein Sci. 1995, 4, 2411-2423.

3. Fialova, M.; Kypr, J.; Vorlickova, M. The Thrombin Binding Aptamer GGTTGGTGTGGTTGG Forms a Bimolecular Guanine Tetraplex. Biochem. Biophys. Res. Commun. 2006, 344, 50-54.

4. Gray, D. M.; Ratliff, R. L.; Vaughan, M. R. Circular Dichroism Spectroscopy of DNA. Methods Enzymol. 1992, 211, 389-406. 